\title{
Solvability for a class of nonlinear Hadamard fractional differential equations with parameters
}

\author{
Meshari Alesemi ${ }^{\text {* }}$
}

\section{"Correspondence:}

malesemi@jazanu.edu.sa

'Department of Mathematics,

Faculty of Science, Jazan University,

Jazan, Saudi Arabia

\begin{abstract}
In this paper, we investigate a class of boundary value problem of nonlinear Hadamard fractional differential equations with $p$-Laplacian operator. By means of the properties of the Green's functions and Guo-Krasnosel'skii fixed point theorem on cones, various existence results for positive solutions are derived in terms of different values of parameters.
\end{abstract}

MSC: $34 \mathrm{~A} 08 ; 34 \mathrm{~B} 15 ; 34 \mathrm{~B} 18 ; 34 \mathrm{~B} 27$

Keywords: Hadamard fractional differential equations; Green's function; Positive solution; $p$-Laplacian operator; Fixed point theorem; Cone

\section{Introduction}

Fractional calculus has emerged as an interesting field of investigation in the last few decades. The subject has been extensively developed and the literature on the topic is much enriched now, covering theoretical as well as widespread applications of this branch of mathematical analysis. In fact, the fractional differential equations have attracted more and more attention for their useful applications in various fields, such as economics, science, and engineering; see [1-5]. In the last few decades, much attention has been focused on the study of the existence of positive solutions for boundary value problems of Riemann-Liouville type or Caputo type fractional differential equations; see [6-23].

On the other hand, $p$-Laplacian operator is extensively applied in the mathematical modeling of several real world phenomena in physics, mechanics, dynamical systems, etc. While studying the fundamental problem of turbulent flow in a porous medium, Leibenson [24] introduced the $p$-Laplacian operator $\phi_{p}(x(t))$ in 1945. Also there has been shown much interest in obtaining the existence and multiplicity of solutions of this class of problems by employing different fixed point theorems. Recently, many scholars have paid more attention to the fractional order differential equation boundary value problems with $p$ Laplacian operator; see [25-32].

The system of fractional differential equations boundary value problems with $p$ Laplacian operator has also received much attention and has developed very rapidly; see [33-39]. In [33], He and Song discussed the following fractional order differential system

(c) The Author(s) 2019. This article is distributed under the terms of the Creative Commons Attribution 4.0 International License (http://creativecommons.org/licenses/by/4.0/), which permits unrestricted use, distribution, and reproduction in any medium, provided you give appropriate credit to the original author(s) and the source, provide a link to the Creative Commons license, and indicate if changes were made. 
with $p$-Laplacian operator:

$$
\left\{\begin{array}{lll}
D_{0^{+}}^{\alpha_{1}}\left(\phi_{p_{1}}\left(D_{0^{+}}^{\beta_{1}} u(t)\right)\right)=\lambda f(t, u(t)), & t \in(0,1), \\
D_{0^{+}}^{\alpha_{2}}\left(\phi_{p_{2}}\left(D_{0^{+}}^{\beta_{2}} u(t)\right)\right)=\mu g(t, u(t)), & t \in(0,1), \\
u(0)=0, \quad u(1)=a_{1} u\left(\xi_{1}\right), & D_{0^{+}}^{\beta_{1}} u(0)=0, & D_{0^{+}}^{\beta_{1}} u(1)=b_{1} D_{0^{+}}^{\beta_{1}} u\left(\eta_{1}\right), \\
v(0)=0, \quad v(1)=a_{1} v\left(\xi_{2}\right), & D_{0^{+}}^{\beta_{2}} \nu(0)=0, & D_{0^{+}}^{\beta_{2}} v(1)=b_{1} D_{0^{+}}^{\beta_{2}} v\left(\eta_{2}\right),
\end{array}\right.
$$

where $\alpha_{i}, \beta_{i} \in(1,2], D_{0^{+}}^{\alpha_{i}}$ and $D_{0^{+}}^{\beta_{i}}$ are the standard Riemann-Liouville derivatives, $\xi_{i}, \eta_{i} \in$ $(0,1), a_{i}, b_{i} \in[0,1], i=1,2, \lambda$ and $\mu$ are positive parameters. The uniqueness of solution was established by using the Banach contraction mapping principle. Hao et al. [34] investigated the existence of positive solutions for a system of nonlinear fractional differential equations nonlocal boundary value problems with parameters and $p$-Laplacian operator,

$$
\left\{\begin{array}{l}
-D_{0^{+}}^{\alpha_{1}}\left(\phi_{p}\left(D_{0^{+}}^{\beta_{1}} u(t)\right)\right)=\lambda f(t, u(t), v(t)), \quad t \in(0,1), \\
-D_{0^{+}}^{\alpha_{2}}\left(\phi_{p}\left(D_{0^{+}}^{\beta_{2}} u(t)\right)\right)=\mu g(t, u(t), v(t)), \quad t \in(0,1), \\
u(0)=u(1)=u^{\prime}(0)=u^{\prime}(1)=0, \quad D_{0^{+}}^{\beta_{1}} u(0)=0, \quad D_{0^{+}}^{\beta_{1}} u(1)=b_{1} D_{0^{+}}^{\beta_{1}} u\left(\eta_{1}\right), \\
v(0)=v(1)=v^{\prime}(0)=v^{\prime}(1)=0, \quad D_{0^{+}}^{\beta_{2}} v(0)=0, \quad D_{0^{+}}^{\beta_{2}} v(1)=b_{1} D_{0^{+}}^{\beta_{2}} v\left(\eta_{2}\right),
\end{array}\right.
$$

where $\alpha_{i} \in(1,2], \beta_{i} \in(3,4] D_{0^{+}}^{\alpha_{i}}$ and $D_{0^{+}}^{\beta_{i}}$ are the standard Riemann-Liouville derivatives, $\eta_{i} \in(0,1), b_{i} \in\left(0, \eta_{i}^{\frac{1-\alpha_{i}}{p_{i}-1}}\right), i=1,2, \lambda$ and $\mu$ are positive parameters.

It has been noticed that most of the above-mentioned work on the topic is based on Riemann-Liouville or Caputo fractional derivatives. In 1892, Hadamard [40] introduced another fractional derivative, which differs from the above-mentioned ones because its definition involves logarithmic function of arbitrary exponent and named the Hadamard derivative. Although many researchers are paying more and more attention to Hadamard type fractional differential equation, the study of the topic is still in its primary stage. For details and recent developments on Hadamard fractional differential equations, see [4148].

From the above review of the literature concerning fractional differential equations, most of the authors investigated only the existence of solutions or positive solutions for Hadamard fractional differential equations without considering the $p$-Laplacian operator. A very few authors established results along with $p$-Laplacian operator, in [47], Wang considered the nonlinear Hadamard fractional differential equation with integral boundary condition and $p$-Laplacian operator

$$
\left\{\begin{array}{l}
D^{\beta}\left(\phi_{p}\left(D^{\alpha} u(t)\right)\right)=f(t, u(t)), \quad t \in(1, T), \\
u(T)=\lambda I^{\alpha} u(\eta), \quad D^{\alpha} u(1)=0, \quad u(1)=0,
\end{array}\right.
$$

where $f$ grows $p-1$ sublinearly at $+\infty$, and by using the Schauder fixed point theorem, a solution existence result is obtain. In [46], Li and Lin used the Guo-Krasnosel'skii fixed point theorem to obtain the existence and uniqueness of positive solutions. We have

$$
\left\{\begin{array}{l}
D^{\beta}\left(\phi_{p}\left(D^{\alpha} u(t)\right)\right)=f(t, u(t)), \quad 1<t<e, \\
u(1)=u^{\prime}(1)=u^{\prime}(e)=0, \quad D^{\alpha} u(1)=D^{\alpha} u(e)=0,
\end{array}\right.
$$


where the continuous function $f:[1, e] \times[0,+\infty) \rightarrow[0,+\infty), 2<\alpha \leq 3,1<\beta \leq 2, D^{\alpha}$ denotes the standard Hadamard fractional derivative of order $\alpha$. Zhang et al. [48] established some existence of positive(nontrivial) solutions for integral boundary conditions of nonlinear Hadamard fractional differential equations with $p$-Laplacian operator.

$$
\left\{\begin{array}{l}
D^{\beta}\left(\phi_{p}\left(D^{\alpha} u(t)\right)\right)=f(t, u(t)), \quad 1<t<e, \\
u(1)=u^{\prime}(1)=u^{\prime}(e)=0, \quad D^{\alpha} u(1)=0, \\
\phi p\left(D^{\alpha} u(e)\right)=\mu \int_{1}^{e} \phi_{p}\left(D^{\alpha} u(t)\right) \frac{d t}{t},
\end{array}\right.
$$

where $\alpha, \beta$, and $\mu$ are three real numbers with $\alpha \in(2,3], \beta \in(1,2]$, and $\mu \in[0, \beta)$, and $f$ is a continuous function on $[1, e] \times \mathbb{R}$.

Motivated by the aforementioned work, we investigate in this paper the existence of positive solutions for the following nonlinear Hadamard fractional differential equation with $p$-Laplacian operator:

$$
\begin{cases}{ }^{H} D_{1^{+}}^{\beta_{1}}\left(\phi_{p}\left({ }^{H} D_{1^{+}}^{\alpha_{1}} u(t)\right)\right)=\lambda^{p-1} f(t, u(t), v(t), w(t)), & t \in(1, e), \\ { }^{H} D_{1^{+}}^{\beta_{2}}\left(\phi_{p}\left({ }^{H} D_{1^{+}}^{\alpha_{2}} v(t)\right)\right)=\mu^{p-1} g(t, u(t), v(t), w(t)), & t \in(1, e), \\ { }^{H} D_{1^{+}}^{\beta_{3}}\left(\phi_{p}\left({ }^{H} D_{1^{+}}^{\alpha_{3}} w(t)\right)\right)=v^{p-1} h(t, u(t), v(t), w(t)), & t \in(1, e),\end{cases}
$$

subject to the three-point boundary conditions

$$
\left\{\begin{array}{l}
u^{(j)}(1)=0, \quad 0 \leq j \leq n-2, \quad \mu_{1} u^{\left(p_{1}\right)}(e)=\lambda_{1} u^{\left(p_{1}\right)}(\xi), \\
\phi_{p}\left({ }^{H} D_{1^{+}}^{\alpha_{1}} u(1)\right)=0=\left({ }^{H} D_{1^{+}}^{p_{2}}\left(\phi_{p}\left({ }^{H} D_{1^{+}}^{\alpha_{1}} u(e)\right)\right)\right), \\
v^{(j)}(1)=0, \quad 0 \leq j \leq m-2, \quad \mu_{1} v^{\left(q_{1}\right)}(e)=\lambda_{1} v^{\left(q_{1}\right)}(\xi), \\
\phi_{p}\left({ }^{H} D_{1^{+}}^{\alpha_{2}} v(1)\right)=0=\left({ }^{H} D_{1^{+}}^{q_{2}}\left(\phi_{p}\left({ }^{H} D_{1^{+}}^{\alpha_{2}} v(e)\right)\right)\right), \\
w^{(j)}(1)=0, \quad 0 \leq j \leq l-2, \quad \mu_{1} w^{\left(r_{1}\right)}(e)=\lambda_{1} w^{\left(r_{1}\right)}(\xi), \\
\phi_{p}\left({ }^{H} D_{1^{+}}^{\alpha_{3}} w(1)\right)=0=\left({ }^{H} D_{1^{+}}^{r_{2}}\left(\phi_{p}\left({ }^{H} D_{1^{+}}^{\alpha_{3}} w(e)\right)\right)\right),
\end{array}\right.
$$

where $\alpha_{i}, \beta_{i} \in \mathbb{R}, i=1,2,3, \alpha_{1} \in(n-1, n], \alpha_{2} \in(m-1, m], \alpha_{3} \in(l-1, l], n, m, l \in \mathbb{N}$ for $n, m, l \geq 3, \beta_{i} \in(1,2], i=1,2,3, p_{1} \in\left[1, \alpha_{1}-1\right), q_{1} \in\left[1, \alpha_{2}-1\right), \gamma_{1} \in\left[1, \alpha_{3}-1\right), p_{2} . q_{2}, \gamma_{2} \in$ $(0,1]$ and $p_{2}, q_{2}, \gamma_{2} \leq \beta_{i}-1, i=1,2,3, \mu_{1}, \lambda_{1} \in(0, \infty), \xi \in(1, e)$ are constants. ${ }^{H} D_{1^{+}}^{k}$ denotes the Hadamard fractional derivative of order $k . \phi_{p}(s)=|s|^{p-2} s, p>1, \phi_{p}^{-1}=\phi_{q}, \frac{1}{p}+\frac{1}{q}=1$, $f, g, h \in C\left([1, e] \times[0,+\infty)^{3},[0,+\infty)\right), \lambda, \mu$ and $v>0$ are positive parameters.

Under some assumptions on $f, g$ and $h$, we give intervals for the parameters $\lambda, \mu$ and $v$ such that positive solutions of (1)-(2) exist. By a positive solution of the problem (1)-(2), we mean a triplet of functions $(u, v, w) \in(C([1, e],[0, \infty)))^{3}$ satisfying (1)-(2) with $u(t)>0$ for all $t \in[1, e]$, or $v(t)>0$ for all $t \in[1, e]$, or $w(t)>0$ for all $t \in[1, e]$ and $(u, v, w) \neq(0,0,0)$.

The main aim of this paper is to investigate the above Hadamard fractional differential equation with $p$-Laplacian operator boundary value problem (1)-(2). With the help of the properties of the Green's functions and the Guo-Krasnosel'skii fixed point theorem on cones, we established the various existence results for positive solutions were derived in terms of different values of $\lambda, \mu$ and $v$, under different combinations of superlinearity and sublinearity of the functions $f, g$ and $h$. At the end, we give an example to illustrate the feasibility of our proposed theoretical result. 


\section{Preliminaries}

For convenience of the reader, we present some necessary definitions and lemmas from Hadamard fractional calculus theory in this section.

Definition 2.1 ([1]) The left-sided Hadamard fractional integrals of order $\alpha \in \mathbb{R}^{+}$of the function $h(t)$ are defined by

$$
\left({ }^{H} I^{\alpha} h\right)(t)=\frac{1}{\Gamma(\alpha)} \int_{1}^{t}\left(\ln \frac{t}{s}\right)^{\alpha-1} h(s) \frac{d s}{s} \quad(1 \leq t \leq e),
$$

where $\Gamma(\cdot)$ is the Gamma function.

Definition 2.2 ([1]) The left-sided Hadamard fractional derivatives of order $\alpha \in(n-1, n]$, $n \in Z^{+}$of the function $h(t)$ are defined by

$$
\left({ }^{H} D^{\alpha} h\right)(t)=\frac{1}{\Gamma(n-\alpha)}\left(t \frac{d}{d t}\right)^{n} \int_{1}^{t}\left(\ln \frac{t}{s}\right)^{n-\alpha+1} h(s) \frac{d s}{s} \quad(1 \leq t \leq e)
$$

where $\Gamma(\cdot)$ is the Gamma function.

Lemma 2.1 ([1]) If $a, \alpha, \beta>0$ then

$$
\left({ }^{H} D_{a}^{\alpha}\left(\ln \frac{t}{a}\right)^{\beta-1}\right)(x)=\frac{\Gamma(\beta)}{\Gamma(\beta-\alpha)}\left(\ln \frac{x}{a}\right)^{\beta-\alpha-1} .
$$

Lemma 2.2 ([1]) Let $q>0$ and $u \in C[1, \infty) \cap L^{1}[1, \infty)$. Then the Hadamard fractional differential equation ${ }^{H} D^{q} u(t)=0$ has the solution

$$
u(t)=\sum_{i=1}^{n} c_{i}(\ln t)^{q-1}
$$

and the following formula holds:

$$
{ }^{H} I^{q H} D^{q} u(t)=u(t)+\sum_{i=1}^{n} c_{i}(\ln t)^{q-i}
$$

where $c_{i} \in R, i=1,2, \ldots, n$ and $n-1<q<n$.

\section{Green's function and bounds}

In this section we present the expression and properties of Green's function associated with boundary value problem (1)-(2). In order to prove our main results, we need some preliminary results.

Lemma 3.1 Let $\Delta_{1}=\mu_{1}-\lambda_{1}(\ln \xi)^{\alpha_{1}-p_{1}-1}>0, h \in C[1, e]$ and $n-1<\alpha_{1} \leq n$, for $n \geq 3$. Then the unique solution of

$$
\left\{\begin{array}{l}
{ }^{H} D_{1^{+}}^{\alpha_{1}} u(t)+h(t)=0, \quad 1<t<e, \\
u^{(j)}(1)=0, \quad 0 \leq j \leq n-2, \quad \mu_{1} u^{\left(p_{1}\right)}(e)=\lambda_{1} u^{\left(p_{1}\right)}(\xi),
\end{array}\right.
$$


is $u(t)=\int_{1}^{e} G_{1}(t, s) h(s) \frac{d s}{s}$, where

$$
\begin{aligned}
& G_{1}(t, s)=G_{11}(t, s)+\frac{\lambda_{1}(\ln t)^{\alpha_{1}-1}}{\mu_{1}-\lambda_{1}(\ln \xi)^{\alpha_{1}-p_{1}-1}} G_{12}(\xi, s), \\
& G_{11}(t, s)=\frac{1}{\Gamma\left(\alpha_{1}\right)} \begin{cases}(\ln t)^{\alpha_{1}-1}(1-\ln s)^{\alpha_{1}-p_{1}-1}-\left(\ln \frac{t}{s}\right)^{\alpha_{1}-1}, & 1 \leq s \leq t \leq e, \\
(\ln t)^{\alpha_{1}-1}(1-\ln s)^{\alpha_{1}-p_{1}-1}, & 1 \leq t \leq s \leq e,\end{cases} \\
& G_{12}(t, s)=\frac{1}{\Gamma\left(\alpha_{1}\right)} \begin{cases}(\ln t)^{\alpha_{1}-p_{1}-1}(1-\ln s)^{\alpha_{1}-p_{1}-1}-\left(\ln \frac{t}{s}\right)^{\alpha_{1}-p_{1}-1}, & 1 \leq s \leq t \leq e, \\
(\ln t)^{\alpha_{1}-p_{1}-1}(1-\ln s)^{\alpha_{1}-p_{1}-1}, & 1 \leq t \leq s \leq e .\end{cases}
\end{aligned}
$$

Proof It is enough to consider the case when $u$ is a solution of (3). From Lemma 2.1 we have ${ }^{H} I_{1^{+}}^{\alpha_{1} H} D_{1^{+}}^{\alpha_{1}} u(t)=-{ }^{H} I_{1^{+}}^{\alpha_{1}} h(t)$, so that

$$
u(t)=-\frac{1}{\Gamma\left(\alpha_{1}\right)} \int_{1}^{e}\left(\ln \frac{t}{s}\right)^{\alpha_{1}-1} h(s) \frac{d s}{s}+c_{1}(\ln t)^{\alpha_{1}-1}+c_{2}(\ln t)^{\alpha_{1}-2}+\cdots+c_{n}(\ln t)^{\alpha_{1}-n},
$$

for some $c_{i} \in \mathbb{R}, i=1,2, \ldots, n$. From $u^{(j)}(1)=0, j=0,1,2, \ldots, n-2$, we have $c_{2}=c_{3}=\cdots=$ $c_{n}=0$. Hence

$$
u^{(j)}(t)=c_{1} \prod_{j=1}^{p_{1}}\left(\alpha_{1}-j\right)(\ln t)^{\alpha_{1}-p_{1}-1}-\prod_{j=1}^{p_{1}}\left(\alpha_{1}-j\right) \frac{1}{\Gamma\left(\alpha_{1}\right)} \int_{1}^{t}\left(\ln \frac{t}{s}\right)^{\alpha_{1}-p_{1}-1} h(s) \frac{d s}{s} .
$$

Then $\mu_{1} u^{\left(p_{1}\right)}(e)=\lambda_{1} u^{\left(p_{1}\right)}(\xi)$ implies that

$$
c_{1}=\frac{1}{\Delta_{1}}\left[\frac{\mu_{1}}{\Gamma\left(\alpha_{1}\right)} \int_{1}^{e}(1-\ln s)^{\alpha_{1}-p_{1}-1} h(s) \frac{d s}{s}-\frac{\lambda_{1}}{\Gamma\left(\alpha_{1}\right)} \int_{1}^{\xi}\left(\ln \frac{\xi}{s}\right)^{\alpha_{1}-p_{1}-1} h(s) \frac{d s}{s}\right] .
$$

As a result,

$$
\begin{aligned}
u(t)= & \frac{-1}{\Gamma\left(\alpha_{1}\right)} \int_{1}^{t}\left(\ln \frac{t}{s}\right)^{\alpha_{1}-1} h(s) \frac{d s}{s}+\frac{\mu_{1}(\ln t)^{\alpha_{1}-1}}{\Delta_{1}} \int_{1}^{e} \frac{(1-\ln s)^{\alpha_{1}-p_{1}-1}}{\Gamma\left(\alpha_{1}\right)} h(s) \frac{d s}{s} \\
& -\frac{\lambda_{1}(\ln t)^{\alpha_{1}-1}}{\Delta_{1}} \int_{1}^{\xi} \frac{\left(\ln \frac{\xi}{s}\right)^{\alpha_{1}-p_{1}-1}}{\Gamma\left(\alpha_{1}\right)} h(s) \frac{d s}{s} \\
= & \frac{-1}{\Gamma\left(\alpha_{1}\right)} \int_{1}^{t}\left(\ln \frac{t}{s}\right)^{\alpha_{1}-1} h(s) \frac{d s}{s}+\frac{(\ln t)^{\alpha_{1}-1}}{\Gamma\left(\alpha_{1}\right)} \int_{1}^{e}(1-\ln s)^{\alpha_{1}-p_{1}-1} h(s) \frac{d s}{s} \\
& -\frac{\lambda_{1}(\ln t)^{\alpha_{1}-1}}{\Delta_{1}} \int_{1}^{\xi} \frac{\left(\ln \frac{\xi}{s}\right)^{\alpha_{1}-p_{1}-1}}{\Gamma\left(\alpha_{1}\right)} h(s) \frac{d s}{s} \\
& +\frac{\lambda_{1}(\ln t)^{\alpha_{1}-1}}{\Delta_{1}} \int_{1}^{e} \frac{(\ln \xi)^{\alpha_{1}-p_{1}-1}(1-\ln s)^{\alpha_{1}-p_{1}-1}}{\Gamma\left(\alpha_{1}\right)} h(s) \frac{d s}{s} \\
= & \int_{1}^{e} G_{1}(t, s) h(s) \frac{d s}{s} .
\end{aligned}
$$

Lemma 3.2 Let $n-1<\alpha_{1} \leq n, 1<\beta_{1} \leq 2$ and $y \in C[1, e]$. Then the unique solution of

$$
\left\{\begin{array}{l}
{ }^{H} D_{1^{+}}^{\beta_{1}}\left(\phi_{p}\left({ }^{H} D_{1^{+}}^{\alpha_{1}} u(t)\right)\right)=y(t), \quad 1<t<e, \\
u^{(j)}(1)=0, \quad 0 \leq j \leq n-2, \quad \mu_{1} u^{\left(p_{1}\right)}(e)=\lambda_{1} u^{\left(p_{1}\right)}(\xi), \\
\phi_{p}\left({ }^{H} D_{1^{+}}^{\alpha_{1}} u(1)\right)=0=\left({ }^{H} D_{1^{+}}^{p_{2}}\left(\phi_{p}\left({ }^{H} D_{1^{+}}^{\alpha_{1}} u(e)\right)\right)\right)
\end{array}\right.
$$


is $u(t)=\int_{1}^{e} G_{1}(t, s) \phi_{q}\left(\int_{1}^{e} H_{1}(s, \tau) y(\tau) \frac{d \tau}{\tau}\right) \frac{d s}{s}$, where $G_{1}(t, s)$ is defined as $(4)$.

$$
H_{1}(t, s)=\frac{1}{\Gamma\left(\beta_{1}\right)} \begin{cases}(\ln t)^{\beta_{1}-1}(1-\ln s)^{\beta_{1}-p_{2}-1}-\left(\ln \frac{t}{s}\right)^{\beta_{1}-1}, & 1 \leq s \leq t \leq e, \\ (\ln t)^{\beta_{1}-1}(1-\ln s)^{\beta_{1}-p_{2}-1}, & 1 \leq t \leq s \leq e .\end{cases}
$$

Proof It is enough to consider the case when $u$ is a solution of (5). From Lemma 2.1 we have

$$
{ }^{H} I_{1^{+}}^{\beta_{1} H} D_{1^{+}}^{\beta_{1}}\left(\phi_{p}\left({ }^{H} D_{1^{+}}^{\alpha_{1}} u(t)\right)\right)=\phi_{p}\left({ }^{H} D_{1^{+}}^{\alpha_{1}} u(t)\right)+c_{1}(\ln t)^{\beta_{1}-1}+c_{2}(\ln t)^{\beta_{1}-2}
$$

for some constants $c_{i} \in \mathbb{R}, i=1,2$. In view of (5), we obtain

$$
{ }^{H} I_{1^{+}}^{\beta_{1} H} D_{1^{+}}^{\beta_{1}}\left(\phi_{p}\left(D_{1^{+}}^{\alpha_{1}} u(t)\right)\right)=I_{1^{+}}^{\beta_{1}} y(t) .
$$

Also we find

$$
\phi_{p}\left({ }^{H} D_{1^{+}}^{\alpha_{1}} u(t)\right)={ }^{H} I_{1^{+}}^{\beta_{1}} y(t)+c_{1}(\ln t)^{\beta_{1}-1}+c_{2}(\ln t)^{\beta_{1}-2} .
$$

Note that $\phi_{p}\left({ }^{H} D_{1^{+}}^{\alpha_{1}} u(1)\right)=0$, we have $c_{2}=0$, then

$$
\begin{aligned}
& { }^{H} D_{1^{+}}^{p_{2}}\left(\phi_{p}\left({ }^{H} D_{1^{+}}^{\alpha_{1}} u(t)\right)\right) \\
& \quad={ }^{H} D_{1^{+}}^{p_{2} H} I_{1^{+}}^{\beta_{1}} y(t)+c_{1}{ }^{H} D_{1^{+}}^{p_{2}}(\ln t)^{\beta_{1}-1} \\
& \quad{ }^{H} I_{1^{+}}^{\left(\beta_{1}-p_{2}\right)} y(t)+c_{1} \frac{\Gamma\left(\beta_{1}\right)}{\Gamma\left(\beta_{1}-p_{2}\right)}(\ln t)^{\beta_{1}-p_{2}-1} \\
& \quad=\frac{1}{\Gamma\left(\beta_{1}-p_{2}\right)} \int_{1}^{t}\left(\ln \frac{t}{s}\right)^{\beta_{1}-p_{2}-1} y(s) \frac{d s}{s}+c_{1} \frac{\Gamma(\beta)}{\Gamma\left(\beta_{1}-p_{2}\right)}(\ln t)^{\beta_{1}-p_{2}-1} .
\end{aligned}
$$

Consequently, $\left({ }^{H} D_{1^{+}}^{p_{2}}\left(\phi_{p}\left({ }^{H} D_{1^{+}}^{\alpha_{1}} u(e)\right)\right)\right)=0$, implies that

$$
c_{1}=\frac{-1}{\Gamma\left(\beta_{1}\right)} \int_{1}^{e}(1-\ln s)^{\beta_{1}-p_{2}-1} y(s) \frac{d s}{s} .
$$

Therefore,

$$
\begin{aligned}
\phi_{p}\left({ }^{H} D_{1^{+}}^{\alpha_{1}} u(t)\right) & =\frac{1}{\Gamma\left(\beta_{1}\right)} \int_{1}^{t}\left(\ln \frac{t}{s}\right)^{\beta_{1}-1} y(s) \frac{d s}{s}-\frac{(\ln t)^{\beta_{1}-1}}{\Gamma\left(\beta_{1}\right)} \int_{1}^{e}(1-\ln s)^{\beta_{1}-p_{2}-1} y(s) \frac{d s}{s} \\
& =-\int_{1}^{e} H_{1}(t, s) y(s) \frac{d s}{s} .
\end{aligned}
$$

Also we have

$$
{ }^{H} D_{1^{+}}^{\alpha_{1}} u(t)+\phi_{q}\left(\int_{1}^{e} H_{1}(t, s) y(s) \frac{d s}{s}\right)=0 .
$$

Noting Lemma 3.1 and the conditions $u^{(j)}(1)=0,0 \leq j \leq n-2, \mu_{1} u^{\left(p_{1}\right)}(e)=\lambda_{1} u^{\left(p_{1}\right)}(\xi)$, we have $u(t)=\int_{1}^{e} G_{1}(t, s) \phi_{q}\left(\int_{1}^{e} H_{1}(t, \tau) y(\tau) \frac{d \tau}{\tau}\right) \frac{d s}{s}$. 
Lemma 3.3 Let $\Delta_{1}>0$. Then the Green's function $G_{1}(t, s)$ given by $(4)$ satisfies the following inequalities:

(i) $G_{1}(t, s) \geq 0$, for all $(t, s) \in[1, e] \times[1, e]$,

(ii) $G_{1}(t, s) \leq G_{1}(e, s)$, for all $(t, s) \in[1, e] \times[1, e]$,

(iii) $G_{1}(t, s) \geq\left(\frac{1}{4}\right)^{\alpha_{1}-1} G_{1}(e, s)$, for all $(t, s) \in\left[e^{1 / 4}, e^{3 / 4}\right] \times(1, e)$.

Proof Consider the Green's function $G_{11}(t, s)$ given by (4).

(i) For $1 \leq t \leq s \leq e$. It is easy to see that $G_{11}(t, s) \geq 0$.

Let $1 \leq s \leq t \leq e$. Then

$$
\begin{aligned}
G_{11}(t, s) & =\frac{1}{\Gamma\left(\alpha_{1}\right)}\left[(\ln t)^{\alpha_{1}-1}(1-\ln s)^{\alpha_{1}-p_{1}-1}-\left(\ln \frac{t}{s}\right)^{\alpha_{1}-1}\right] \\
& =\frac{1}{\Gamma\left(\alpha_{1}\right)}\left[(\ln t)^{\alpha_{1}-1}(1-\ln s)^{\alpha_{1}-p_{1}-1}-\left(1-\frac{\ln s}{\ln t}\right)^{\alpha_{1}-1}(\ln t)^{\alpha_{1}-1}\right] \\
& \geq \frac{(\ln t)^{\alpha_{1}-1}}{\Gamma\left(\alpha_{1}\right)}\left[(1-\ln s)^{\alpha_{1}-p_{1}-1}-(1-\ln s)^{\alpha_{1}-1}\right] \\
& =\frac{(\ln t)^{\alpha_{1}-1}}{\Gamma\left(\alpha_{1}\right)}\left[p_{1} \ln s+\frac{p_{1}\left(p_{1}+1\right)}{2}(\ln s)^{2}+\cdots\right](1-\ln s)^{\alpha_{1}-1} \geq 0 \\
& =\frac{(\ln t)^{\alpha_{1}-1}}{\Gamma\left(\alpha_{1}\right)}\left[p_{1} \ln s+O(\ln s)^{2}\right](1-\ln s)^{\alpha_{1}-1} \geq 0 .
\end{aligned}
$$

On the other hand, if $1 \leq s \leq \xi \leq e$,

$$
\begin{aligned}
G_{12}(\xi, s) & =\frac{1}{\Gamma\left(\alpha_{1}\right)}\left[(\ln \xi)^{\alpha_{1}-p_{1}-1}(1-\ln s)^{\alpha_{1}-p_{1}-1}-\left(\ln \frac{\xi}{s}\right)^{\alpha_{1}-p_{1}-1}\right] \\
& =\frac{1}{\Gamma\left(\alpha_{1}\right)}\left[(\ln \xi)^{\alpha_{1}-p_{1}-1}(1-\ln s)^{\alpha_{1}-p_{1}-1}-\left(1-\frac{\ln s}{\ln \xi}\right)^{\alpha_{1}-p_{1}-1}(\ln \xi)^{\alpha_{1}-p_{1}-1}\right] \\
& \geq \frac{(\ln \xi)^{\alpha_{1}-p_{1}-1}}{\Gamma\left(\alpha_{1}\right)}\left[(1-\ln s)^{\alpha_{1}-p_{1}-1}-(1-\ln s)^{\alpha_{1}-p_{1}-1}\right]=0,
\end{aligned}
$$

which implies $G_{1}(t, s) \geq 0$. Hence the inequality (i) is proved.

(ii) For $1 \leq t \leq s \leq e$. It is easy to see that $\frac{d G_{11}(t, s)}{d t} \geq 0$.

Let $1 \leq s \leq \xi \leq e$. Then we have

$$
\begin{aligned}
\frac{d G_{11}(t, s)}{d t}= & \frac{\left(\alpha_{1}-1\right)}{\Gamma\left(\alpha_{1}\right)}\left[(\ln t)^{\alpha_{1}-2}(1-\ln s)^{\alpha_{1}-p_{1}-1}-\left(\ln \frac{t}{s}\right)^{\alpha_{1}-2}\right] \\
\geq & \frac{\left(\alpha_{1}-1\right)(\ln t)^{\alpha_{1}-2}}{\Gamma\left(\alpha_{1}\right)}\left[(1-\ln s)^{\alpha_{1}-p_{1}-1}-(1-\ln s)^{\alpha_{1}-2}\right] \\
= & \frac{\left(\alpha_{1}-1\right)(\ln t)^{\alpha_{1}-2}}{\Gamma\left(\alpha_{1}\right)}\left[1-\left(1-\left(p_{1}-1\right) \ln s+\frac{\left(p_{1}-1\right)\left(p_{1}-2\right)}{2}(\ln s)^{2}\right.\right. \\
& +\cdots](1-\ln s)^{\alpha_{1}-p_{1}-1} \\
= & \frac{\left(\alpha_{1}-1\right)(\ln t)^{\alpha_{1}-2}}{\Gamma\left(\alpha_{1}\right)}\left[\left(p_{1}-1\right) \ln s+O(\ln s)^{2}\right](1-\ln s)^{\alpha_{1}-p_{1}-1} \geq 0 .
\end{aligned}
$$

On the other hand, consider $\xi \leq s$. It is easy to see that $G_{12}(\xi, s) \geq 0$. 
For $s \leq \xi$ one has

$$
\begin{aligned}
& (\ln \xi)^{\alpha_{1}-p_{1}-1}(1-\ln s)^{\alpha_{1}-p_{1}-1}-\left(\ln \frac{\xi}{s}\right)^{\alpha_{1}-p_{1}-1} \\
& \quad \geq(\ln \xi)^{\alpha_{1}-p_{1}-1}(1-\ln s)^{\alpha_{1}-p_{1}-1}-(\ln \xi-\ln \xi \ln s)^{\alpha_{1}-p_{1}-1} \geq 0 .
\end{aligned}
$$

Therefore

$$
\frac{d G_{1}(t, s)}{d t}=\frac{d G_{11}(t, s)}{d t}+\frac{\lambda_{1}\left(\alpha_{1}-1\right)(\ln t)^{\alpha_{1}-2}}{\mu_{1}-\lambda_{1}(\ln \xi)^{\alpha_{1}-p_{1}-1}} G_{12}(\xi, s) \geq 0
$$

which implies that $G_{1}(t, s)$ is the monotone nondecreasing function, so

$$
G_{1}(t, s) \leq G_{1}(e, s) \quad \text { for all }(t, s) \in[1, e] \times[1, e]
$$

Hence the inequality (ii) is proved.

(iii) For $1 \leq t \leq s \leq e$,

$$
\frac{G_{11}(t, s)}{G_{11}(e, s)}=\frac{(\ln t)^{\alpha-1}(1-\ln s)^{\alpha-\beta-1}}{(1-\ln s)^{\alpha-\beta-1}}=(\ln t)^{\alpha-1}
$$

For $1 \leq s \leq t \leq e$,

$$
\begin{aligned}
\frac{G_{11}(t, s)}{G_{11}(e, s)} & =\frac{(\ln t)^{\alpha_{1}-1}(1-\ln s)^{\alpha_{1}-p_{1}-1}-\left(\ln \frac{t}{s}\right)^{\alpha_{1}-1}}{(1-\ln s)^{\alpha_{1}-p_{1}-1}-(1-\ln s)^{\alpha_{1}-1}} \\
& \geq \frac{(\ln t)^{\alpha_{1}-1}(1-\ln s)^{\alpha_{1}-p_{1}-1}-(\ln t-\ln s \ln t)^{\alpha_{1}-1}}{(1-\ln s)^{\alpha_{1}-p_{1}-1}-(1-\ln s)^{\alpha_{1}-1}} \\
& =\frac{(\ln t)^{\alpha_{1}-1}\left[(1-\ln s)^{\alpha_{1}-p_{1}-1}-(1-\ln s)^{\alpha_{1}-1}\right]}{(1-\ln s)^{\alpha_{1}-p_{1}-1}-(1-\ln s)^{\alpha_{1}-1}}=(\ln t)^{\alpha_{1}-1} .
\end{aligned}
$$

Therefore

$$
G_{11}(t, s) \geq(\ln t)^{\alpha_{1}-1} G_{11}(e, s) \quad \text { for all }(t, s) \in[1, e] \times(1, e) .
$$

From (4) and (7) we have

$$
\begin{aligned}
G_{1}(t, s) & =G_{11}(t, s)+\frac{\lambda_{1}(\ln t)^{\alpha_{1}-1}}{\mu_{1}-\lambda_{1}(\ln \xi)^{\alpha_{1}-p_{1}-1}} G_{12}(\xi, s) \\
& \geq(\ln t)^{\alpha_{1}-1} G_{11}(e, s)+\frac{\lambda_{1}(\ln t)^{\alpha_{1}-1}}{\mu_{1}-\lambda_{1}(\ln \xi)^{\alpha_{1}-p_{1}-1}} G_{12}(\xi, s) \\
& =(\ln t)^{\alpha_{1}-1} G_{1}(e, s) \geq\left(\frac{1}{4}\right)^{\alpha_{1}-1} G_{1}(e, s) \quad \text { for all }(t, s) \in\left[e^{1 / 4}, e^{3 / 4}\right] \times(1, e)
\end{aligned}
$$

Therefore $G_{1}(t, s) \geq\left(\frac{1}{4}\right)^{\alpha_{1}-1} G_{1}(e, s)$. Hence the inequality (iii) is proved.

Lemma 3.4 Let $\Delta_{1}>0$. Then the Green's function $H_{1}(t, s)$ given by (6) satisfies the following inequalities:

(i) $0 \leq H_{1}(t, s) \leq H_{1}(s, s)$, for all $(t, s) \in[1, e] \times[1, e]$, 
(ii) $H_{1}(t, s) \geq \delta_{1}(s) H_{1}(s, s)$, for all $(t, s) \in\left[e^{1 / 4}, e^{3 / 4}\right] \times(1, e)$,

$$
\delta_{1}(s)= \begin{cases}\frac{\left(\frac{3}{4}\right)^{\beta_{1}-1}(1-\ln s)^{\beta_{1}-p_{2}-1}-\left(\frac{3}{4}-\ln s\right)^{\beta_{1}-1}}{(\ln s)^{\beta_{1}-1}(1-\ln s)^{\beta_{1}-p_{2}-1}} H_{1}(s, s), & s \in(1, r], \\ \frac{1}{(4 \ln s)^{\beta-1}} H_{1}(s, s), & s \in[r, e) .\end{cases}
$$

Proof (i) For $1 \leq t \leq s \leq e$, it is easy to show that $\frac{d}{d t} H_{1}(t, s) \geq 0$ for all $(t, s) \in[1, e] \times[1, e]$, then

$$
0=H_{1}(1, s) \leq H_{1}(t, s) \leq H_{1}(s, s) \text { for all } t \leq s
$$

Let $s \leq t$. Then

$$
\begin{aligned}
\frac{d}{d t} H_{1}(t, s) & =\frac{1}{\Gamma\left(\beta_{1}\right)}\left[\left(\beta_{1}-1\right)(\ln t)^{\beta_{1}-2}(1-\ln s)^{\beta_{1}-p_{2}-1}-\left(\beta_{1}-1\right)\left(\ln \frac{t}{s}\right)^{\beta_{1}-2}\right] \\
& \leq \frac{\left(\beta_{1}-1\right)}{\Gamma\left(\beta_{1}\right)}\left[(1-\ln s)^{\beta_{1}-p_{2}-1}-(1-\ln s)^{\beta_{1}-2}\right] \\
& =\frac{\left(\beta_{1}-1\right)}{\Gamma\left(\beta_{1}\right)}\left[(1-\ln s)^{-p_{2}+1}-1\right](1-\ln s)^{\beta_{1}-2} \\
& =\frac{\left(\beta_{1}-1\right)}{\Gamma\left(\beta_{1}\right)}\left[\left(p_{2}-1\right) \ln s+\frac{\left(p_{2}-1\right)\left(p_{2}\right)}{2 !}(\ln s)^{2}+\cdots\right](1-\ln s)^{\beta_{1}-2} \\
& =\frac{\left(\beta_{1}-1\right)}{\Gamma\left(\beta_{1}\right)}\left[\left(p_{2}-1\right) \ln s+O(\ln s)^{2}\right](1-\ln s)^{\beta_{1}-2} \leq 0 .
\end{aligned}
$$

Therefore, $H_{1}(t, s)$ is decreasing in $t$ for $s \in[1, e]$, which implies that $H_{1}(t, s) \leq H_{1}(s, s)$. Hence, the inequality (i) is proved.

(ii) For $s \in(1, e) . H_{1}(t, s)$ is increasing in $t$ for $t \leq s$ and decreasing in $t$ for $s \leq t$. We define

$$
\begin{aligned}
& h_{11}(t, s)=\frac{1}{\Gamma\left(\beta_{1}\right)}\left[(\ln t)^{\beta_{1}-1}(1-\ln s)^{\beta_{1}-p_{2}-1}-\left(\ln \frac{t}{s}\right)^{\beta_{1}-1}\right], \\
& h_{12}(t, s)=\frac{1}{\Gamma\left(\beta_{1}\right)}\left[(\ln t)^{\beta_{1}-1}(1-\ln s)^{\beta_{1}-p_{2}-1}\right],
\end{aligned}
$$

and applying the monotonicity of $H_{1}(t, s)$, we have

$$
\begin{aligned}
& \min _{e^{1 / 4} \leq t \leq e^{3 / 4}} H_{1}(t, s)= \begin{cases}h_{11}\left(e^{3 / 4}, s\right), & s \in\left(1, e^{1 / 4}\right], \\
\min \left\{h_{1}\left(e^{3 / 4}, s\right), h_{12}\left(e^{1 / 4}, s\right)\right\}, & s \in\left[e^{1 / 4}, e^{3 / 4}\right], \\
h_{12}\left(e^{1 / 4}, s\right), & s \in\left[e^{3 / 4}, e\right),\end{cases} \\
& = \begin{cases}h_{11}\left(e^{3 / 4}, s\right), & s \in(1, r), \\
h_{12}\left(e^{1 / 4}, s\right), & s \in[r, e)\end{cases} \\
& = \begin{cases}\frac{1}{\Gamma\left(\beta_{1}\right)}\left[\left(\frac{3}{4}\right)^{\beta_{1}-1}(1-\ln s)^{\beta_{1}-p_{2}-1}-\left(\frac{3}{4}-\ln s\right)^{\beta_{1}-1}\right], & s \in(1, r], \\
\frac{1}{\Gamma\left(\beta_{1}\right)}\left[\left(\frac{1}{4}\right)^{\beta_{1}-1}(1-\ln s)^{\beta_{1}-p_{2}-1}\right], & s \in[r, e),\end{cases}
\end{aligned}
$$




$$
\begin{aligned}
& \geq \begin{cases}\frac{\left(\frac{3}{4}\right)^{\beta_{1}-1}(1-\ln s)^{\beta_{1}-p_{2}-1}-\left(\frac{3}{4}-\ln s\right)^{\beta_{1}-1}}{(\ln s)^{\beta_{1}-1}(1-\ln s)^{\beta_{1}-p_{2}-1}} H_{1}(s, s), & s \in(1, r], \\
\frac{1}{(4 \ln s)^{\beta_{1}-1}} H_{1}(s, s), & s \in[r, e),\end{cases} \\
& =\delta_{1}(s) H_{1}(s, s) \quad \text { for } s \in(1, e) .
\end{aligned}
$$

Hence, the inequality (ii) is proved.

We can also formulate similar results to Lemmas 3.1-3.4 for the Hadamard fractional boundary value problems

$$
\left\{\begin{array}{l}
{ }^{H} D_{1^{+}}^{\beta_{2}}\left(\phi_{p}\left({ }^{H} D_{1^{+}}^{\alpha_{2}} v(t)\right)\right)=\mu^{p-1} g(t, u(t), v(t), w(t)), \quad t \in(1, e) \\
v^{(j)}(1)=0, \quad 0 \leq j \leq m-2, \quad \mu_{1} v^{\left(q_{1}\right)}(e)=\lambda_{1} v^{\left(q_{1}\right)}(\xi) \\
\phi_{p}\left({ }^{H} D_{1^{+}}^{\alpha_{2}} v(1)\right)=0=\left({ }^{H} D_{1^{+}}^{q_{2}}\left(\phi_{p}\left({ }^{H} D_{1^{+}}^{\alpha_{2}} v(e)\right)\right)\right)
\end{array}\right.
$$

and

$$
\left\{\begin{array}{l}
{ }^{H} D_{1^{+}}^{\beta_{3}}\left(\phi_{p}\left({ }^{H} D_{1^{+}}^{\alpha_{3}} w(t)\right)\right)=v^{p-1} h(t, u(t), v(t), w(t)), \quad t \in(1, e), \\
w^{(j)}(1)=0, \quad 0 \leq j \leq l-2, \quad \mu_{1} w^{\left(r_{1}\right)}(e)=\lambda_{1} w^{\left(r_{1}\right)}(\xi), \\
\phi_{p}\left({ }^{H} D_{1^{+}}^{\alpha_{3}} w(1)\right)=0=\left({ }^{H} D_{1^{+}}^{r_{2}}\left(\phi_{p}\left({ }^{H} D_{1^{+}}^{\alpha_{3}} w(e)\right)\right)\right) .
\end{array}\right.
$$

Remark In a similar manner, the results of the Green's functions $G_{2}(t, s), G_{3}(t, s), H_{2}(t, s)$ and $H_{3}(t, s)$ for the homogeneous BVP corresponding to the Hadamard fractional differential equations (8) and (9) are obtained.

Consider the following conditions:

(i) $G_{i}(t, s) \geq m G_{i}(e, s)$, for all $(t, s) \in[1, e] \times[1, e], i=1.2 .3$,

(ii) $H_{i}(t, s) \geq \delta(s) H_{i}(e, s)$, for all $(t, s) \in I \times(1, e), i=1,2,3$,

where $I=\left[e^{1 / 4}, e^{3 / 4}\right], m=\min \left\{\left(\frac{1}{4}\right)^{\alpha_{1}-1},\left(\frac{1}{4}\right)^{\alpha_{2}-1},\left(\frac{1}{4}\right)^{\alpha_{3}-1}\right\}, \delta(s)=\min \left\{\delta_{1}(s), \delta_{2}(s), \delta_{3}(s)\right\}$.

Our main results are based on the following Guo-Krasnosel'skii fixed point theorem on cones.

Theorem 3.5 (Krasnosel'skii $[49,50])$ Let $X$ be a Banach space, $K \subseteq X$ be a cone, and suppose that $\Omega_{1}, \Omega_{2}$ are open subsets of $X$ with $0 \in \Omega_{1}$ and $\bar{\Omega}_{1} \subset \Omega_{2}$. Suppose furthermore that $T: K \cap\left(\bar{\Omega}_{2} \backslash \Omega_{1}\right) \rightarrow K$ is a completely continuous operator such that either

(i) $\|T u\| \leq\|u\|, u \in K \cap \partial \Omega_{1}$ and $\|T u\| \geq\|u\|, u \in K \cap \partial \Omega_{2}$, or

(ii) $\|T u\| \geq\|u\|, u \in K \cap \partial \Omega_{1}$ and $\|T u\| \leq\|u\|, u \in K \cap \partial \Omega_{2}$,

holds. Then $T$ has a fixed point in $K \cap\left(\bar{\Omega}_{2} \backslash \Omega_{1}\right)$.

\section{Positive solutions in a cone}

In this section, we shall give sufficient conditions on $\lambda, \mu, v, f, g$ and $h$ such that positive solutions with respect to a cone for our problem (1)-(2) exist.

We present the assumptions that we shall use in the sequel:

(A1) The functions $f, g, h:[1, e] \times[0,+\infty)^{3} \rightarrow[0,+\infty)$ are continuous.

(A2) $\alpha_{i}, \beta_{i} \in \mathbb{R}, i=1,2,3, \alpha_{1} \in(n-1, n], \alpha_{2} \in(m-1, m], \alpha_{3} \in(l-1, l], n, m, l \in \mathbb{N}$ for $n, m, l \geq 3, \beta_{i} \in(1,2], i=1,2,3, p_{1} \in\left[1, \alpha_{1}-1\right), q_{1} \in\left[1, \alpha_{2}-1\right), \gamma_{1} \in\left[1, \alpha_{3}-1\right)$, 
$p_{2} . q_{2}, \gamma_{2} \in(0,1], p_{2}, q_{2}, \gamma_{2} \leq \beta_{i}-1, i=1,2,3, \mu_{1}, \lambda_{1} \in(0, \infty), \xi \in(1, e)$ are constants and $\Delta_{1}=\mu_{1}-\lambda_{1}(\ln \xi)^{\alpha_{1}-p_{1}-1}>0, \Delta_{2}=\mu_{1}-\lambda_{1}(\ln \xi)^{\alpha_{2}-q_{1}-1}>0, \Delta_{3}=\mu_{1}-$ $\lambda_{1}(\ln \xi)^{\alpha_{3}-r_{1}-1}>0$.

(A3) For $I=\left[e^{1 / 4}, e^{3 / 4}\right] \subset(1, e)$, we introduce the following extreme limits:

$$
\begin{array}{ll}
f_{0}^{s}=\limsup _{u+v+w \rightarrow 0} \max _{t \in[1, e]} \frac{f(t, u, v, w)}{(u+v+w)^{p-1}}, \quad g_{0}^{s}=\limsup _{u+v+w \rightarrow 0} \max _{t \in[1, e]} \frac{g(t, u, v, w)}{(u+v+w)^{p-1}}, \\
h_{0}^{s}=\limsup _{u+v+w \rightarrow 0} \max _{t \in[1, e]} \frac{h(t, u, v, w)}{(u+v+w)^{p-1}}, \quad f_{0}^{i}=\liminf _{u+v+w \rightarrow 0} \min _{t \in I} \frac{f(t, u, v, w)}{(u+v+w)^{p-1}}, \\
g_{0}^{i}=\liminf _{u+v+w \rightarrow 0} \min _{t \in I} \frac{g(t, u, v, w)}{(u+v+w)^{p-1}}, & h_{0}^{i}=\liminf _{u+v+w \rightarrow 0} \min _{t \in I} \frac{h(t, u, v, w)}{(u+v+w)^{p-1}}, \\
f_{\infty}^{s}=\limsup _{u+v+w \rightarrow \infty} \max _{t \in[1, e]} \frac{f(t, u, v, w)}{(u+v+w)^{p-1}}, & g_{\infty}^{s}=\limsup _{u+v+w \rightarrow \infty} \max _{t \in[1, e]} \frac{g(t, u, v, w)}{(u+v+w)^{p-1}}, \\
h_{\infty}^{s}=\limsup _{u+v+w \rightarrow \infty} \max _{t \in[1, e]} \frac{h(t, u, v, w)}{(u+v+w)^{p-1}}, & f_{\infty}^{i}=\liminf _{u+v+w \rightarrow \infty} \min _{t \in I} \frac{f(t, u, v, w)}{(u+v+w)^{p-1}}, \\
g_{\infty}^{i}=\liminf _{u+v+w \rightarrow \infty} \max _{t \in I} \frac{g(t, u, v, w)}{(u+v+w)^{p-1}}, & h_{\infty}^{i}=\liminf _{u+v+w \rightarrow \infty} \min _{t \in I} \frac{h(t, u, v, w)}{(u+v+w)^{p-1}} .
\end{array}
$$

Let $X=C[1, e]$, then $X$ is a Banach space with the norm $\|u\|=\sup _{t \in[1, e]}|u(t)|$. Let $Y=$ $X \times X \times X$, then $Y$ is a Banach space with the norm $\|(u, v, w)\|_{Y}=\|u\|+\|v\|+\|w\|$.

Define a cone $P \subset Y$ by

$$
\begin{aligned}
P= & \{(u, v, w) \in Y: u(t) \geq 0, v(t) \geq 0, w(t) \geq 0, \forall t \in[1, e], \\
& \left.\min _{t \in I}\{u(t)+v(t)+w(t)\} \geq m\|(u, v, w)\|_{Y}\right\},
\end{aligned}
$$

where $I=\left[e^{1 / 4}, e^{3 / 4}\right]$. For $\lambda, \mu, v>0$, we define now the operator $Q: P \rightarrow Y$ by $Q(u, v, w)=$ $\left(Q_{1}(u, v, w), Q_{2}(u, v, w), Q_{3}(u, v, w)\right)$ with

$$
\begin{aligned}
& Q_{1}(u, v, w)(t)=\lambda \int_{1}^{e} G_{1}(t, s)\left(\int_{1}^{e} H_{1}(s, \tau) f(\tau, u(\tau), v(\tau), w(\tau)) \frac{d \tau}{\tau}\right)^{\frac{1}{p-1}} \frac{d s}{s}, \quad t \in[1, e] \\
& Q_{2}(u, v, w)(t)=\mu \int_{1}^{e} G_{2}(t, s)\left(\int_{1}^{e} H_{2}(s, \tau) g(\tau, u(\tau), v(\tau), w(\tau)) \frac{d \tau}{\tau}\right)^{\frac{1}{p-1}} \frac{d s}{s}, \quad t \in[1, e] \\
& Q_{3}(u, v, w)(t)=v \int_{1}^{e} G_{3}(t, s)\left(\int_{1}^{e} H_{3}(s, \tau) h(\tau, u(\tau), v(\tau), w(\tau)) \frac{d \tau}{\tau}\right)^{\frac{1}{p-1}} \frac{d s}{s}, \quad t \in[1, e] .
\end{aligned}
$$

Lemma 4.1 $Q: P \rightarrow P$ is a completely continuous operator.

Proof The continuity of functions $G_{i}(t, s), H_{i}(t, s), i=1,2,3$ and $f, g, h$ implies that $Q: P \rightarrow$ $P$ is continuous. For all $(t, s) \in I \times[1, e]$, where $I=\left[e^{1 / 4}, e^{3 / 4}\right]$, we have

$$
\begin{aligned}
\min _{t \in I}\left\{Q_{1}(u, v, w)(t)+Q_{2}(u, v, w)(t)+Q_{3}(u, v, w)(t)\right\} \\
=\min _{t \in I}\left\{\lambda \int_{1}^{e} G_{1}(t, s)\left(\int_{1}^{e} H_{1}(s, \tau) f(\tau, u(\tau), v(\tau), w(\tau)) \frac{d \tau}{\tau}\right)^{\frac{1}{p-1}} \frac{d s}{s}\right. \\
\quad+\mu \int_{1}^{e} G_{2}(t, s)\left(\int_{1}^{e} H_{2}(s, \tau) g(\tau, u(\tau), v(\tau), w(\tau)) \frac{d \tau}{\tau}\right)^{\frac{1}{p-1}} \frac{d s}{s}
\end{aligned}
$$




$$
\begin{aligned}
& \left.+v \int_{1}^{e} G_{3}(t, s)\left(\int_{1}^{e} H_{3}(s, \tau) h(\tau, u(\tau), v(\tau), w(\tau)) \frac{d \tau}{\tau}\right)^{\frac{1}{p-1}} \frac{d s}{s}\right\} \\
\geq & m\left\{\lambda \int_{1}^{e} G_{1}(e, s)\left(\int_{1}^{e} H_{1}(s, \tau) f(\tau, u(\tau), v(\tau), w(\tau)) \frac{d \tau}{\tau}\right)^{\frac{1}{p-1}} \frac{d s}{s}\right. \\
& +\mu \int_{1}^{e} G_{2}(e, s)\left(\int_{1}^{e} H_{2}(s, \tau) g(\tau, u(\tau), v(\tau), w(\tau)) \frac{d \tau}{\tau}\right)^{\frac{1}{p-1}} \frac{d s}{s} \\
& \left.+v \int_{1}^{e} G_{3}(e, s)\left(\int_{1}^{e} H_{3}(s, \tau) h(\tau, u(\tau), v(\tau), w(\tau)) \frac{d \tau}{\tau}\right)^{\frac{1}{p-1}} \frac{d s}{s}\right\} \\
\geq & m\left(\left\|Q_{1}(u, v, w)\right\|+\left\|Q_{2}(u, v, w)\right\|+\left\|Q_{3}(u, v, w)\right\|\right) \\
= & m\left\|\left(Q_{1}(u, v, w), Q_{2}(u, v, w), Q_{3}(u, v, w)\right)\right\| \\
= & m\|Q(u, v, w)\| .
\end{aligned}
$$

Thus $Q(P) \subset P$. So, we can easily show that $Q: P \rightarrow P$ is a completely continuous operator by the Arzela-Ascoli theorem.

If $(u, v, w) \in P$ is a fixed point of operator $Q$, then $(u, v, w)$ is a solution of problem (1)-(2). So, we will investigate the existence of fixed points of operator $Q$.

First, for $f_{0}^{s}, g_{0}^{s}, h_{0}^{s} f_{\infty}^{i}, g_{\infty}^{i}, h_{\infty}^{i} \in(0, \infty)$ and positive numbers $\sigma_{1}, \sigma_{2}, \sigma_{3}>0$ such that $\sigma_{1}+$ $\sigma_{2}+\sigma_{3}=1$, we define the positive numbers $L_{1}, L_{2}, L_{3}, L_{4}, L_{5}$ and $L_{6}$ by

$$
\begin{aligned}
& L_{1}=\sigma_{1}\left[m^{2} \int_{t \in I} G_{1}(e, s)\left(\int_{t \in I} \delta(\tau) H_{1}(\tau, \tau) f_{\infty}^{i} \frac{d \tau}{\tau}\right)^{\frac{1}{p-1}} \frac{d s}{s}\right]^{-1}, \\
& L_{2}=\sigma_{1}\left[\int_{1}^{e} G_{1}(e, s)\left(\int_{1}^{e} H_{1}(s, \tau) f_{0}^{s} \frac{d \tau}{\tau}\right)^{\frac{1}{p-1}} \frac{d s}{s}\right]^{-1}, \\
& L_{3}=\sigma_{2}\left[m^{2} \int_{t \in I} G_{2}(e, s)\left(\int_{t \in I} \delta(\tau) H_{2}(\tau, \tau) g_{\infty}^{i} \frac{d \tau}{\tau}\right)^{\frac{1}{p-1}} \frac{d s}{s}\right]^{-1}, \\
& L_{4}=\sigma_{2}\left[\int_{1}^{e} G_{2}(e, s)\left(\int_{1}^{e} H_{2}(s, \tau) g_{0}^{s} \frac{d \tau}{\tau}\right)^{\frac{1}{p-1}} \frac{d s}{s}\right]^{-1}, \\
& L_{5}=\sigma_{3}\left[m^{2} \int_{t \in I} G_{3}(e, s)\left(\int_{t \in I} \delta(\tau) H_{3}(\tau, \tau) h_{\infty}^{i} \frac{d \tau}{\tau}\right)^{\frac{1}{p-1}} \frac{d s}{s}\right]^{-1}, \\
& L_{6}=\sigma_{3}\left[\int_{1}^{e} G_{3}(e, s)\left(\int_{1}^{e} H_{3}(s, \tau) h_{0}^{s} \frac{d \tau}{\tau}\right)^{\frac{1}{p-1}} \frac{d s}{s}\right]^{-1} \cdot
\end{aligned}
$$

Theorem 4.2 Assume that (A1)-(A3) hold, $\sigma_{1}, \sigma_{2}, \sigma_{3}>0$ with $\sigma_{1}+\sigma_{2}+\sigma_{3}=1$.

(a) If $f_{0}^{s}, g_{0}^{s}, h_{0}^{s}, f_{\infty}^{i}, g_{\infty}^{i}, h_{\infty}^{i} \in(0, \infty), L_{1}<L_{2}, L_{3}<L_{4}$ and $L_{5}<L_{6}$, then for each $\lambda \in\left(L_{1}, L_{2}\right), \mu \in\left(L_{3}, L_{4}\right)$ and $v \in\left(L_{5}, L_{6}\right)$ there exists a positive solution $(u(t), v(t), w(t)), t \in[1, e]$, for problem (1)-(2).

(b) If $f_{0}^{s}=g_{0}^{s}=h_{0}^{s}=0, f_{\infty}^{i}, g_{\infty}^{i}, h_{\infty}^{i} \in(0, \infty)$, then for each $\lambda \in\left(L_{1}, \infty\right), \mu \in\left(L_{3}, \infty\right)$ and $v \in\left(L_{5}, \infty\right)$ there exists a positive solution $(u(t), v(t), w(t)), t \in[1, e]$, for problem (1)-(2). 
(c) If $f_{0}^{s}, g_{0}^{s}, h_{0}^{s} \in(0, \infty), f_{\infty}^{i}=g_{\infty}^{i}=h_{\infty}^{i}=\infty$ then for each $\lambda \in\left(0, L_{2}\right), \mu \in\left(0, L_{4}\right)$ and $v \in\left(0, L_{6}\right)$ there exists a positive solution $(u(t), v(t), w(t)), t \in[1, e]$, for problem (1)-(2).

(d) If $f_{0}^{s}=g_{0}^{s}=h_{0}^{s}=0, f_{\infty}^{i}=g_{\infty}^{i}=h_{\infty}^{i}=\infty$, then for each $\lambda \in(0, \infty), \mu \in(0, \infty)$ and $v \in(0, \infty)$ there exists a positive solution $(u(t), v(t), w(t)), t \in[1, e]$, for problem (1)-(2).

Proof Because the proofs of these cases are similar, in what follows we will prove two of them, namely cases (a) and (b).

(a) For any $\lambda \in\left(L_{1}, L_{2}\right), \mu \in\left(L_{3}, L_{4}\right), v \in\left(L_{5}, L_{6}\right)$ let $\epsilon>0$ be a positive number such that $0<\epsilon<\min \left\{f_{\infty}^{i}, g_{\infty}^{i}, h_{\infty}^{i}\right\}$ and

$$
\begin{aligned}
& \sigma_{1}\left[m^{2}\left(f_{\infty}^{i}-\epsilon\right)^{\frac{1}{p-1}} \int_{t \in I} G_{1}(e, s)\left(\int_{t \in I} \delta(\tau) H_{1}(\tau, \tau) \frac{d \tau}{\tau}\right)^{\frac{1}{p-1}} \frac{d s}{s}\right]^{-1} \leq \lambda, \\
& \sigma_{1}\left[\left(f_{0}^{s}+\epsilon\right)^{\frac{1}{p-1}} \int_{1}^{e} G_{1}(e, s)\left(\int_{1}^{e} H_{1}(s, \tau) \frac{d \tau}{\tau}\right)^{\frac{1}{p-1}} \frac{d s}{s}\right]^{-1} \geq \lambda, \\
& \sigma_{2}\left[m^{2}\left(g_{\infty}^{i}-\epsilon\right)^{\frac{1}{p-1}} \int_{t \in I} G_{2}(e, s)\left(\int_{t \in I} \delta(\tau) H_{2}(\tau, \tau) \frac{d \tau}{\tau}\right)^{\frac{1}{p-1}} \frac{d s}{s}\right]^{-1} \leq \mu, \\
& \sigma_{2}\left[\left(g_{0}^{s}+\epsilon\right)^{\frac{1}{p-1}} \int_{1}^{e} G_{2}(e, s)\left(\int_{1}^{e} H_{2}(s, \tau) \frac{d \tau}{\tau}\right)^{\frac{1}{p-1}} \frac{d s}{s}\right]^{-1} \geq \mu \\
& \sigma_{3}\left[m^{2}\left(h_{\infty}^{i}-\epsilon\right)^{\frac{1}{p-1}} \int_{t \in I} G_{3}(e, s)\left(\int_{t \in I} \delta(\tau) H_{3}(\tau, \tau) \frac{d \tau}{\tau}\right)^{\frac{1}{p-1}} \frac{d s}{s}\right]^{-1} \leq v, \\
& \sigma_{3}\left[\left(h_{0}^{s}+\epsilon\right)^{\frac{1}{p-1}} \int_{1}^{e} G_{3}(e, s)\left(\int_{1}^{e} H_{3}(s, \tau) \frac{d \tau}{\tau}\right)^{\frac{1}{p-1}} \frac{d s}{s}\right]^{-1} \geq v .
\end{aligned}
$$

By the definitions of $f_{0}^{s}, g_{0}^{s}$ and $h_{0}^{s}$ there exists $R_{1}>0$ such that

$$
\begin{array}{ll}
f(t, u, v, w) \leq\left(f_{0}^{s}+\epsilon\right)(u+v+w)^{p-1}, & t \in[1, e], 0 \leq u+v+w \leq R_{1}, \\
g(t, u, v, w) \leq\left(g_{0}^{s}+\epsilon\right)(u+v+w)^{p-1}, & t \in[1, e], 0 \leq u+v+w \leq R_{1}, \\
h(t, u, v, w) \leq\left(h_{0}^{s}+\epsilon\right)(u+v+w)^{p-1}, & t \in[1, e], 0 \leq u+v+w \leq R_{1} .
\end{array}
$$

Let $(u, v, w) \in P$ with $\|(u, v, w)\|_{Y}=R_{1}$ i.e. $\|u\|+\|v\|+\|w\|=R_{1}$, then we have

$$
\begin{aligned}
Q_{1}(u, v, w)(t) & =\lambda \int_{1}^{e} G_{1}(t, s)\left(\int_{1}^{e} H_{1}(s, \tau) f(\tau, u(\tau), v(\tau), w(\tau)) \frac{d \tau}{\tau}\right)^{\frac{1}{p-1}} \frac{d s}{s} \\
& \leq \lambda \int_{1}^{e} G_{1}(e, s)\left(\int_{1}^{e} H_{1}(s, \tau)\left(f_{0}^{s}+\epsilon\right)(u(\tau)+v(\tau)+w(\tau))^{p-1} \frac{d \tau}{\tau}\right)^{\frac{1}{p-1}} \frac{d s}{s} \\
& \leq \lambda\left(f_{0}^{s}+\epsilon\right)^{\frac{1}{p-1}} \int_{1}^{e} G_{1}(e, s)\left(\int_{1}^{e} H_{1}(s, \tau)(\|u\|+\|v\|+\|w\|)^{p-1} \frac{d \tau}{\tau}\right)^{\frac{1}{p-1}} \frac{d s}{s} \\
& \leq \sigma_{1}\|(u, v, w)\|_{Y}, \quad \text { for all } t \in[1, e],
\end{aligned}
$$




$$
\begin{aligned}
Q_{2}(u, v, w)(t) & =\mu \int_{1}^{e} G_{2}(t, s)\left(\int_{1}^{e} H_{2}(s, \tau) g(\tau, u(\tau), v(\tau), w(\tau)) \frac{d \tau}{\tau}\right)^{\frac{1}{p-1}} \frac{d s}{s} \\
& \leq \mu \int_{1}^{e} G_{2}(e, s)\left(\int_{1}^{e} H_{2}(s, \tau)\left(g_{0}^{s}+\epsilon\right)(u(\tau)+v(\tau)+w(\tau))^{p-1} \frac{d \tau}{\tau}\right)^{\frac{1}{p-1}} \frac{d s}{s} \\
& \leq \mu\left(g_{0}^{s}+\epsilon\right)^{\frac{1}{p-1}} \int_{1}^{e} G_{2}(e, s)\left(\int_{1}^{e} H_{2}(s, \tau)(\|u\|+\|v\|+\|w\|)^{p-1} \frac{d \tau}{\tau}\right)^{\frac{1}{p-1}} \frac{d s}{s} \\
& \leq \sigma_{2}\|(u, v, w)\|_{Y}, \quad \text { for all } t \in[1, e], \\
Q_{3}(u, v, w)(t) & =v \int_{1}^{e} G_{3}(t, s)\left(\int_{1}^{e} H_{3}(s, \tau) h(\tau, u(\tau), v(\tau), w(\tau)) \frac{d \tau}{\tau}\right)^{\frac{1}{p-1}} \frac{d s}{s} \\
& \leq v \int_{1}^{e} G_{3}(e, s)\left(\int_{1}^{e} H_{3}(s, \tau)\left(h_{0}^{s}+\epsilon\right)(u(\tau)+v(\tau)+w(\tau))^{p-1} \frac{d \tau}{\tau}\right)^{\frac{1}{p-1}} \frac{d s}{s} \\
& \leq v\left(h_{0}^{s}+\epsilon\right)^{\frac{1}{p-1}} \int_{1}^{e} G_{3}(e, s)\left(\int_{1}^{e} H_{3}(s, \tau)(\|u\|+\|v\|+\|w\|)^{p-1} \frac{d \tau}{\tau}\right)^{\frac{1}{p-1}} \frac{d s}{s} \\
& \leq \sigma_{3}\|(u, v, w)\|_{Y}, \quad \text { for all } t \in[1, e] .
\end{aligned}
$$

Therefore,

$$
\begin{aligned}
\|Q(u, v, w)\|_{Y} & =\left\|\left(Q_{1}(u, v, w), Q_{2}(u, v, w), Q_{3}(u, v, w)\right)\right\|_{Y} \\
& =\left\|Q_{1}(u, v, w)\right\|+\left\|Q_{2}(u, v, w)\right\|+\left\|Q_{3}(u, v, w)\right\| \\
& \leq \sigma_{1}\|(u, v, w)\|_{Y}+\sigma_{2}\|(u, v, w)\|_{Y}+\sigma_{3}\|(u, v, w)\|_{Y} \\
& =\left(\sigma_{1}+\sigma_{2}+\sigma_{3}\right)\|(u, v, w)\|_{Y} \\
& =\|(u, v, w)\|_{Y} .
\end{aligned}
$$

Hence, $\|Q(u, v, w)\|_{Y} \leq\|(u, v, w)\|_{Y}$. Define the set

$$
\Omega_{1}=\left\{(u, v, w) \in Y:\|(u, v, w)\|_{Y}<R_{1}\right\}
$$

then

$$
\|Q(u, v, w)\|_{Y} \leq\|(u, v, w)\|_{Y}, \quad \text { for }(u, v, w) \in P \cap \partial \Omega_{1} .
$$

On the other hand, by the definitions of $f_{\infty}^{i}, g_{\infty}^{i}$ and $h_{\infty}^{i}$, there exists $\bar{R}_{2}>0$ such that

$$
\begin{array}{ll}
f(t, u, v) \geq\left(f_{\infty}^{i}-\epsilon\right)(u+v+w)^{p-1}, & t \in I, u, v, w \geq 0, u+v+w \geq \bar{R}_{2}, \\
g(t, u, v) \geq\left(g_{\infty}^{i}-\epsilon\right)(u+v+w)^{p-1}, & t \in I, u, v, w \geq 0, u+v+w \geq \bar{R}_{2}, \\
h(t, u, v) \geq\left(h_{\infty}^{i}-\epsilon\right)(u+v+w)^{p-1}, & t \in I, u, v, w \geq 0, u+v+w \geq \bar{R}_{2},
\end{array}
$$

Let $R_{2}=\max \left\{2 R_{1}, \frac{\overline{R_{2}}}{m}\right\}$. Choose $(u, v, w) \in P$ with $\|(u, v, w)\|_{Y}=R_{2}$. Then

$$
\min _{t \in I}(u(t)+v(t)+w(t)) \geq m\|(u, v, w)\|_{Y}=m R_{2} \geq \bar{R}_{2} .
$$


Also we have

$$
\begin{aligned}
& Q_{1}(u, v, w)(t) \\
& =\lambda \int_{1}^{e} G_{1}(t, s)\left(\int_{1}^{e} H_{1}(s, \tau) f(\tau, u(\tau), v(\tau), w(\tau)) \frac{d \tau}{\tau}\right)^{\frac{1}{p-1}} \frac{d s}{s} \\
& \geq \lambda m \int_{t \in I} G_{1}(e, s)\left(\int_{1}^{e} H_{1}(s, \tau)\left(f_{\infty}^{i}-\epsilon\right)(u(\tau)+v(\tau)+w(\tau))^{p-1} \frac{d \tau}{\tau}\right)^{\frac{1}{p-1}} \frac{d s}{s} \\
& \geq \lambda m^{2}\left(f_{\infty}^{i}-\epsilon\right)^{\frac{1}{p-1}} \int_{t \in I} G_{1}(e, s)\left(\int_{t \in I} \delta(\tau) H_{1}(\tau, \tau)\left(\|(u, v, w)\|_{Y}\right)^{p-1} \frac{d \tau}{\tau}\right)^{\frac{1}{p-1}} \frac{d s}{s} \\
& \geq \sigma_{1}\|(u, v, w)\|_{Y}, \\
& Q_{2}(u, v, w)(t) \\
& =\lambda \int_{1}^{e} G_{2}(t, s)\left(\int_{1}^{e} H_{2}(s, \tau) g(\tau, u(\tau), v(\tau), w(\tau)) \frac{d \tau}{\tau}\right)^{\frac{1}{p-1}} \frac{d s}{s} \\
& \geq \mu m \int_{t \in I} G_{2}(e, s)\left(\int_{1}^{e} H_{2}(s, \tau)\left(g_{\infty}^{i}-\epsilon\right)(u(\tau)+v(\tau)+w(\tau))^{p-1} \frac{d \tau}{\tau}\right)^{\frac{1}{p-1}} \frac{d s}{s} \\
& \geq \mu m^{2}\left(g_{\infty}^{i}-\epsilon\right)^{\frac{1}{p-1}} \int_{t \in I} G_{2}(e, s)\left(\int_{t \in I} \delta(\tau) H_{2}(\tau, \tau)\left(\|(u, v, w)\|_{Y}\right)^{p-1} \frac{d \tau}{\tau}\right)^{\frac{1}{p-1}} \frac{d s}{s} \\
& \geq \sigma_{2}\|(u, v, w)\|_{Y}, \\
& Q_{3}(u, v, w)(t) \\
& =\lambda \int_{1}^{e} G_{3}(t, s)\left(\int_{1}^{e} H_{3}(s, \tau) h(\tau, u(\tau), v(\tau), w(\tau)) \frac{d \tau}{\tau}\right)^{\frac{1}{p-1}} \frac{d s}{s} \\
& \geq v m \int_{s \in I} G_{3}(e, s)\left(\int_{1}^{e} H_{3}(s, \tau)\left(h_{\infty}^{i}-\epsilon\right)(u(\tau)+v(\tau)+w(\tau))^{p-1} \frac{d \tau}{\tau}\right)^{\frac{1}{p-1}} \frac{d s}{s} \\
& \geq v m^{2}\left(h_{\infty}^{i}-\epsilon\right)^{\frac{1}{p-1}} \int_{t \in I} G_{3}(e, s)\left(\int_{\tau \in I} \delta(\tau) H_{3}(\tau, \tau)\left(\|(u, v, w)\|_{Y}\right)^{p-1} \frac{d \tau}{\tau}\right)^{\frac{1}{p-1}} \frac{d s}{s} \\
& \geq \sigma_{3}\|(u, v, w)\|_{Y} .
\end{aligned}
$$

Therefore,

$$
\begin{aligned}
\|Q(u, v, w)\|_{Y} & =\left\|\left(Q_{1}(u, v, w), Q_{2}(u, v, w), Q_{3}(u, v, w)\right)\right\|_{Y} \\
& =\left\|Q_{1}(u, v, w)\right\|+\left\|Q_{2}(u, v, w)\right\|+\left\|Q_{3}(u, v, w)\right\| \\
& \geq \sigma_{1}\|(u, v, w)\|_{Y}+\sigma_{2}\|(u, v, w)\|_{Y}+\sigma_{3}\|(u, v, w)\|_{Y} \\
& =\left(\sigma_{1}+\sigma_{2}+\sigma_{3}\right)\|(u, v, w)\|_{Y} \\
& =\|(u, v, w)\|_{Y} .
\end{aligned}
$$

Hence, $\|Q(u, v, w)\|_{Y} \geq\|(u, v, w)\|_{Y}$. Define the set

$$
\Omega_{2}=\left\{(u, v, w) \in Y:\|(u, v, w)\|_{Y}<R_{2}\right\}
$$


then

$$
\|Q(u, v, w)\|_{Y} \geq\|(u, v, w)\|_{Y}, \quad \text { for }(u, v, w) \in P \cap \partial \Omega_{2}
$$

Therefore, by (10), (11) and Theorem 3.5, we conclude that $Q$ has at least one fixed point $(u, v, w) \in P \cap\left(\bar{\Omega}_{2} \backslash \Omega_{1}\right)$ with $R_{1} \leq\|(u, v, w)\|_{Y} \leq R_{2}$.

(b) Let $\lambda \in\left(L_{1}, \infty\right), \mu \in\left(L_{3}, \infty\right), v \in\left(L_{5}, \infty\right)$ and let $\epsilon>0$ be a positive number such that $\epsilon<f_{\infty}^{i}, \epsilon<g_{\infty}^{i}, \epsilon<h_{\infty}^{i}$. We have

$$
\begin{aligned}
& \sigma_{1}\left[m^{2}\left(f_{\infty}^{i}-\epsilon\right)^{\frac{1}{p-1}} \int_{t \in I} G_{1}(e, s)\left(\int_{t \in I} \delta(\tau) H_{1}(\tau, \tau) \frac{d \tau}{\tau}\right)^{\frac{1}{p-1}} \frac{d s}{s}\right]^{-1} \leq \lambda, \\
& \sigma_{2}\left[m^{2}\left(g_{\infty}^{i}-\epsilon\right)^{\frac{1}{p-1}} \int_{t \in I} G_{2}(e, s)\left(\int_{t \in I} \delta(\tau) H_{2}(\tau, \tau) \frac{d \tau}{\tau}\right)^{\frac{1}{p-1}} \frac{d s}{s}\right]^{-1} \leq \mu, \\
& \sigma_{3}\left[m^{2}\left(h_{\infty}^{i}-\epsilon\right)^{\frac{1}{p-1}} \int_{t \in I} G_{3}(e, s)\left(\int_{t \in I} \delta(\tau) H_{3}(\tau, \tau) \frac{d \tau}{\tau}\right)^{\frac{1}{p-1}} \frac{d s}{s}\right]^{-1} \leq v \\
& \epsilon \leq \frac{\sigma_{1}}{\lambda}\left[\int_{1}^{e} G_{1}(e, s)\left(\int_{1}^{e} H_{1}(s, \tau) \frac{d \tau}{\tau}\right)^{\frac{1}{p-1}} \frac{d s}{s}\right]^{-1}, \\
& \epsilon \leq \frac{\sigma_{2}}{\mu}\left[\int_{1}^{e} G_{2}(e, s)\left(\int_{1}^{e} H_{2}(s, \tau) \frac{d \tau}{\tau}\right)^{\frac{1}{p-1}} \frac{d s}{s}\right]^{-1}, \\
& \epsilon \leq \frac{\sigma_{3}}{v}\left[\int_{1}^{e} G_{3}(e, s)\left(\int_{1}^{e} H_{3}(s, \tau) \frac{d \tau}{\tau}\right)^{\frac{1}{p-1}} \frac{d s}{s}\right]^{-1} .
\end{aligned}
$$

By the definitions of $f_{0}^{s}=0, g_{0}^{s}=0$ and $h_{0}^{s}=0$, there exists $R_{1}>0$ such that

$$
\begin{array}{ll}
f(t, u, v, w) \leq \epsilon^{p-1}(u+v+w)^{p-1}, & 0 \leq u+v+w \leq R_{1}, \\
g(t, u, v, w) \leq \epsilon^{p-1}(u+v+w)^{p-1}, & 0 \leq u+v+w \leq R_{1}, \\
h(t, u, v, w) \leq \epsilon^{p-1}(u+v+w)^{p-1}, & 0 \leq u+v+w \leq R_{1} .
\end{array}
$$

Let $(u, v, w) \in P$ with $\|(u, v, w)\|_{Y}=R_{1}$ i.e. $\|u\|+\|v\|+\|w\|=R_{1}$. Then we have

$$
\begin{aligned}
Q_{1}(u, v, w)(t) & =\lambda \int_{1}^{e} G_{1}(t, s)\left(\int_{1}^{e} H_{1}(s, \tau) f(\tau, u(\tau), v(\tau), w(\tau)) \frac{d \tau}{\tau}\right)^{\frac{1}{p-1}} \frac{d s}{s} \\
& \leq \lambda \int_{1}^{e} G_{1}(e, s)\left(\int_{1}^{e} H_{1}(s, \tau) \epsilon^{p-1}(u(\tau)+v(\tau)+w(\tau))^{p-1} \frac{d \tau}{\tau}\right)^{\frac{1}{p-1}} \frac{d s}{s} \\
& \leq \lambda \epsilon \int_{1}^{e} G_{1}(e, s)\left(\int_{1}^{e} H_{1}(s, \tau)(\|u\|+\|v\|+\|w\|)^{p-1} \frac{d \tau}{\tau}\right)^{\frac{1}{p-1}} \frac{d s}{s} \\
& \leq \sigma_{1}\|(u, v, w)\|_{Y}, \quad \text { for all } t \in[1, e] .
\end{aligned}
$$

Hence, $\left\|Q_{1}(u, v, w)\right\| \leq \sigma_{1}\|(u, v, w)\|_{Y}$. In a similar manner, we conclude that

$$
\left\|Q_{2}(u, v, w)\right\| \leq \sigma_{2}\|(u, v, w)\|_{Y^{\prime}}, \quad\left\|Q_{3}(u, v, w)\right\| \leq \sigma_{3}\|(u, v, w)\|_{Y} .
$$


Therefore,

$$
\begin{aligned}
\|Q(u, v, w)\|_{Y} & =\left\|\left(Q_{1}(u, v, w), Q_{2}(u, v, w), Q_{3}(u, v, w)\right)\right\|_{Y} \\
& =\left\|Q_{1}(u, v, w)\right\|+\left\|Q_{2}(u, v, w)\right\|+\left\|Q_{3}(u, v, w)\right\| \\
& \leq \sigma_{1}\|(u, v, w)\|_{Y}+\sigma_{2}\|(u, v, w)\|_{Y}+\sigma_{3}\|(u, v, w)\|_{Y} \\
& =\left(\sigma_{1}+\sigma_{2}+\sigma_{3}\right)\|(u, v, w)\|_{Y} \\
& =\|(u, v, w)\|_{Y} .
\end{aligned}
$$

Hence, $\|Q(u, v, w)\|_{Y} \leq\|(u, v, w)\|_{Y}$. Define the set

$$
\Omega_{1}=\left\{(u, v, w) \in Y:\|(u, v, w)\|_{Y}<R_{1}\right\}
$$

then

$$
\|Q(u, v, w)\|_{Y} \leq\|(u, v, w)\|_{Y}, \quad \text { for }(u, v, w) \in P \cap \partial \Omega_{1} .
$$

By the definitions of $f_{\infty}^{i}, g_{\infty}^{i}, h_{\infty}^{i} \in(0, \infty)$, there exists $\bar{R}_{2}>0$ such that

$$
\begin{array}{ll}
f(t, u, v, w) \geq\left(f_{\infty}^{i}-\epsilon\right)(u+v+w)^{p-1}, & u+v+w \geq \bar{R}_{2}, \\
g(t, u, v, w) \geq\left(g_{\infty}^{i}-\epsilon\right)(u+v+w)^{p-1}, & u+v+w \geq \bar{R}_{2}, \\
h(t, u, v, w) \geq\left(h_{\infty}^{i}-\epsilon\right)(u+v+w)^{p-1}, & u+v+w \geq \bar{R}_{2} .
\end{array}
$$

Define the set

$$
\Omega_{2}=\left\{(u, v, w) \in Y \mid\|(u, v, w)\|_{Y}<R_{2}\right\}
$$

and proceeding in a similar manner of proof (a), we get

$$
\|Q(u, v, w)\|_{Y} \geq\|(u, v, w)\|_{Y}, \quad \text { for }(u, v, w) \in P \cap \partial \Omega_{2} .
$$

Therefore, by (12), (13) and Theorem 3.5, we conclude that $Q$ has at least one fixed point $(u, v, w) \in P \cap\left(\bar{\Omega}_{2} \backslash \Omega_{1}\right)$ with $R_{1} \leq\|(u, v, w)\|_{Y} \leq R_{2}$. Similarly, we can prove the remaining.

Prior to our next result, we define the positive numbers $M_{1}, M_{2}, M_{3}, M_{4}, M_{5}$ and $M_{6}$ by

$$
\begin{aligned}
& M_{1}=\rho_{1}\left[m^{2}\left(f_{0}^{i}-\epsilon\right)^{\frac{1}{p-1}} \int_{s \in I} G_{1}(e, s)\left(\int_{\tau \in I} \delta(\tau) H_{1}(\tau, \tau) \frac{d \tau}{\tau}\right)^{\frac{1}{p-1}} \frac{d s}{s}\right]^{-1}, \\
& M_{2}=\rho_{1}\left[\left(f_{\infty}^{s}+\epsilon\right)^{\frac{1}{p-1}} \int_{1}^{e} G_{1}(e, s)\left(\int_{1}^{e} H_{1}(\tau, \tau) \frac{d \tau}{\tau}\right)^{\frac{1}{p-1}} \frac{d s}{s}\right]^{-1}, \\
& M_{3}=\rho_{2}\left[m^{2}\left(g_{0}^{i}-\epsilon\right)^{\frac{1}{p-1}} \int_{s \in I} G_{2}(e, s)\left(\int_{\tau \in I} \delta(\tau) H_{2}(\tau, \tau) \frac{d \tau}{\tau}\right)^{\frac{1}{p-1}} \frac{d s}{s}\right]^{-1}, \\
& M_{4}=\rho_{2}\left[\left(g_{\infty}^{s}+\epsilon\right)^{\frac{1}{p-1}} \int_{1}^{e} G_{2}(e, s)\left(\int_{1}^{e} H_{2}(\tau, \tau) \frac{d \tau}{\tau}\right)^{\frac{1}{p-1}} \frac{d s}{s}\right]^{-1},
\end{aligned}
$$




$$
\begin{aligned}
& M_{5}=\rho_{3}\left[m^{2}\left(h_{0}^{i}-\epsilon\right)^{\frac{1}{p-1}} \int_{s \in I} G_{3}(e, s)\left(\int_{\tau \in I} \delta(\tau) H_{3}(\tau, \tau) \frac{d \tau}{\tau}\right)^{\frac{1}{p-1}} \frac{d s}{s}\right]^{-1}, \\
& M_{6}=\rho_{3}\left[\left(h_{\infty}^{s}+\epsilon\right)^{\frac{1}{p-1}} \int_{1}^{e} G_{3}(e, s)\left(\int_{1}^{e} H_{3}(\tau, \tau) \frac{d \tau}{\tau}\right)^{\frac{1}{p-1}} \frac{d s}{s}\right]^{-1},
\end{aligned}
$$

where $\rho_{1}, \rho_{2}, \rho_{3}>0$ are three positive numbers with $\rho_{1}+\rho_{2}+\rho_{3}=1$.

Theorem 4.3 Assume that the conditions (A1)-(A3) hold.

(a) If $f_{0}^{i}, g_{0}^{i}, h_{0}^{i} f_{\infty}^{s}, g_{\infty}^{s}, h_{\infty}^{s} \in(0, \infty), M_{1}<M_{2}, M_{3}<M_{4}$ and $M_{5}<M_{6}$ then for each $\lambda \in\left(M_{1}, M_{2}\right), \mu \in\left(M_{3}, M_{4}\right)$ and $v \in\left(M_{5}, M_{6}\right)$ there exists a positive solution $(u(t), v(t), w(t)), t \in[1, e]$, for problem $(1)-(2)$.

(b) If $f_{\infty}^{s}=g_{\infty}^{s}=h_{\infty}^{s}=0, f_{0}^{i}, g_{0}^{i}, h_{0}^{i} \in(0, \infty)$, then for each $\lambda \in\left(M_{1}, \infty\right), \mu \in\left(M_{3}, \infty\right)$ and $v \in\left(M_{5}, \infty\right)$ there exists a positive solution $(u(t), v(t), w(t)), t \in[1, e]$, for problem (1) $-(2)$.

(c) If $f_{\infty}^{s}, g_{\infty}^{s}, h_{\infty}^{s} \in(0, \infty), f_{0}^{i}=g_{0}^{i}=h_{0}^{i}=\infty$ then for each $\lambda \in\left(0, M_{2}\right), \mu \in\left(0, M_{4}\right)$ and $v \in\left(0, M_{6}\right)$ there exists a positive solution $(u(t), v(t), w(t)), t \in[1, e]$, for problem (1) $-(2)$.

(d) If $f_{\infty}^{s}=g_{\infty}^{s}=h_{\infty}^{s}=0, f_{0}^{i}=g_{0}^{i}=h_{0}^{i}=\infty$, then for each $\lambda \in(0, \infty), \mu \in(0, \infty)$ and $v \in(0, \infty)$ there exists a positive solution $(u(t), v(t), w(t)), t \in[1, e]$, for problem (1)-(2).

Proof (a) For any $\lambda \in\left(M_{1}, M_{2}\right), \mu \in\left(M_{3}, M_{4}\right), v \in\left(M_{5}, M_{6}\right)$, there exists $0<\epsilon<\min \left\{f_{0}^{i}\right.$, $\left.g_{0}^{i}, h_{0}^{i}\right\}$ such that

$$
\begin{aligned}
& \rho_{1}\left[m^{2}\left(f_{0}^{i}-\epsilon\right)^{\frac{1}{p-1}} \int_{s \in I} G_{1}(e, s)\left(\int_{\tau \in I} \delta(\tau) H_{1}(\tau, \tau) \frac{d \tau}{\tau}\right)^{\frac{1}{p-1}} \frac{d s}{s}\right]^{-1} \leq \lambda, \\
& \rho_{1}\left[\left(f_{\infty}^{s}+\epsilon\right)^{\frac{1}{p-1}} \int_{1}^{e} G_{1}(e, s)\left(\int_{1}^{e} H_{1}(\tau, \tau) \frac{d \tau}{\tau}\right)^{\frac{1}{p-1}} \frac{d s}{s}\right]^{-1} \geq \lambda, \\
& \rho_{2}\left[m^{2}\left(g_{0}^{i}-\epsilon\right)^{\frac{1}{p-1}} \int_{s \in I} G_{2}(e, s)\left(\int_{\tau \in I} \delta(\tau) H_{2}(\tau, \tau) \frac{d \tau}{\tau}\right)^{\frac{1}{p-1}} \frac{d s}{s}\right]^{-1} \leq \mu, \\
& \rho_{2}\left[\left(g_{\infty}^{s}+\epsilon\right)^{\frac{1}{p-1}} \int_{1}^{e} G_{2}(e, s)\left(\int_{1}^{e} H_{2}(\tau, \tau) \frac{d \tau}{\tau}\right)^{\frac{1}{p-1}} \frac{d s}{s}\right]^{-1} \geq \mu, \\
& \rho_{3}\left[m^{2}\left(h_{0}^{i}-\epsilon\right)^{\frac{1}{p-1}} \int_{s \in I} G_{3}(e, s)\left(\int_{\tau \in I} \delta(\tau) H_{3}(\tau, \tau) \frac{d \tau}{\tau}\right)^{\frac{1}{p-1}} \frac{d s}{s}\right]^{-1} \leq v, \\
& \rho_{3}\left[\left(h_{\infty}^{s}+\epsilon\right)^{\frac{1}{p-1}} \int_{1}^{e} G_{3}(e, s)\left(\int_{1}^{e} H_{3}(\tau, \tau) \frac{d \tau}{\tau}\right)^{\frac{1}{p-1}} \frac{d s}{s}\right]^{-1} \geq v .
\end{aligned}
$$

By the definitions of $f_{0}^{i}, g_{0}^{i} \in(0, \infty)$ and $h_{0}^{i} \in(0, \infty)$, we deduce that there exists $R_{3}>0$ such that

$$
\begin{array}{ll}
f(t, u, v, w) \geq\left(f_{0}^{i}-\epsilon\right)(u+v+w)^{p-1}, & t \in I, u, v, w \geq 0, u+v+w \leq R_{3}, \\
g(t, u, v, w) \geq\left(g_{0}^{i}-\epsilon\right)(u+v+w)^{p-1}, & t \in I, u, v, w \geq 0, u+v+w \leq R_{3} \\
h(t, u, v, w) \geq\left(h_{0}^{i}-\epsilon\right)(u+v+w)^{p-1}, & t \in I, u, v, w \geq 0, u+v+w \leq R_{3} .
\end{array}
$$


Let $(u, v, w) \in P$ with $\|(u, v, w)\|_{Y}=R_{3}$, that is, $\|u\|+\|v\|+\|w\|=R_{3}$. Because $u(t)+v(t)+$ $w(t) \leq\|u\|+\|v\|+\|w\|=R_{3}$ for all $t \in[1 . e]$, we have

$$
\begin{aligned}
& Q_{1}(u, v, w)(t) \\
& \quad=\lambda \int_{1}^{e} G_{1}(t, s)\left(\int_{1}^{e} H_{1}(s, \tau) f(\tau, u(\tau), v(\tau), w(\tau)) \frac{d \tau}{\tau}\right)^{\frac{1}{p-1}} \frac{d s}{s} \\
& \quad \geq \lambda m \int_{s \in I} G_{1}(e, s)\left(\int_{\tau \in I} \delta_{1}(\tau) H_{1}(\tau, \tau) f(\tau, u(\tau), v(\tau), w(\tau)) \frac{d \tau}{\tau}\right)^{\frac{1}{p-1}} \frac{d s}{s} \\
& \quad \geq \lambda m \int_{s \in I} G_{1}(e, s)\left(\int_{\tau \in I} H_{1}(\tau, \tau)\left(f_{0}^{i}-\epsilon\right)(u(\tau)+v(\tau)+w(\tau))^{p-1} \frac{d \tau}{\tau}\right)^{\frac{1}{p-1}} \frac{d s}{s} \\
& \quad \geq \lambda m^{2}\left(f_{0}^{i}-\epsilon\right)^{\frac{1}{p-1}} \int_{s \in I} G_{1}(e, s)\left(\int_{\tau \in I} \delta(\tau) H_{1}(\tau, \tau) \frac{d \tau}{\tau}\right)^{\frac{1}{p-1}} \frac{d s}{s}(\|u\|+\|v\|+\|w\|) \\
& \quad \geq \rho_{1}\|(u, v, w)\| .
\end{aligned}
$$

Hence, $\left\|Q_{1}(u, v, w)\right\| \geq \rho_{1}\|(u, v, w)\|$. In a similar manner, we conclude that $\left\|Q_{2}(u, v, w)\right\| \geq$ $\rho_{2}\|(u, v, w)\|,\left\|Q_{3}(u, v, w)\right\| \geq \rho_{3}\|(u, v, w)\|$. Therefore,

$$
\begin{aligned}
\|Q(u, v, w)\|_{Y} & =\left\|\left(Q_{1}(u, v, w), Q_{2}(u, v, w), Q_{3}(u, v, w)\right)\right\|_{Y} \\
& =\left\|Q_{1}(u, v, w)\right\|+\left\|Q_{2}(u, v, w)\right\|+\left\|Q_{3}(u, v, w)\right\| \\
& \geq \rho_{1}\|(u, v, w)\|_{Y}+\rho_{2}\|(u, v, w)\|_{Y}+\rho_{3}\|(u, v, w)\|_{Y} \\
& =\left(\rho_{1}+\rho_{2}+\rho_{3}\right)\|(u, v, w)\|_{Y} \\
& =\|(u, v, w)\|_{Y} .
\end{aligned}
$$

Hence, $\|Q(u, v, w)\|_{Y} \geq\|(u, v, w)\|_{Y}$. Define the set

$$
\Omega_{3}=\left\{(u, v, w) \in Y:\|(u, v, w)\|_{Y}<R_{3}\right\}
$$

then

$$
\|Q(u, v, w)\|_{Y} \geq\|(u, v, w)\|_{Y}, \quad \text { for }(u, v, w) \in P \cap \partial \Omega_{3} .
$$

On the other hand, we define $f^{\star}, g^{\star} \cdot h^{\star}:[1, e] \times \mathbb{R}^{+} \times \mathbb{R}^{+} \times \mathbb{R}^{+} \rightarrow \mathbb{R}^{+}$as follows:

$$
\begin{aligned}
f^{\star}(t, x) & =\max _{0 \leq u+v+w \leq x} f(t, u, v, w), \\
g^{\star}(t, x) & =\max _{0 \leq u+v+w \leq x} g(t, u, v), \\
h^{\star}(t, x) & =\max _{0 \leq u+v+w \leq x} h(t, u, v), \quad \text { for all } x \in \mathbb{R}^{+} .
\end{aligned}
$$

Then

$$
f(t, u, v, w) \leq f^{\star}(t, x), g(t, u, v, w) \leq g^{\star}(t, x), h(t, u, v, w) \leq h^{\star}(t, x), \quad u+v+w \leq x .
$$


It follows that the functions $f^{\star}, g^{\star}, h^{\star}$ are nondecreasing and satisfy the conditions

$$
\begin{aligned}
& \limsup _{x \rightarrow+\infty} \max _{t \in[1, e]} \frac{f^{\star}(t, x)}{x^{p-1}} \leq f_{\infty}^{s}, \\
& \limsup _{x \rightarrow+\infty} \max _{t \in[1, e]} \frac{g^{\star}(t, x)}{x^{p-1}} \leq g_{\infty}^{s}, \\
& \limsup _{x \rightarrow+\infty} \max _{t \in[1, e]} \frac{h^{\star}(t, x)}{x^{p-1}} \leq h_{\infty}^{s} .
\end{aligned}
$$

Next, by the definitions of $f_{\infty}^{s}, g_{\infty}^{s}, h_{\infty}^{s} \in(0, \infty)$, there exists $\bar{R}_{4}>0$ such that, for any $t \in$ $[1, e], x \geq \bar{R}_{4}$, we have

$$
\begin{aligned}
f^{\star}(t, x) & \leq\left(f_{\infty}^{s}+\epsilon\right) x^{p-1}, \quad g^{\star}(t, x) \leq\left(g_{\infty}^{s}+\epsilon\right) x^{p-1}, \\
h^{\star}(t, x) & \leq\left(h_{\infty}^{s}+\epsilon\right) x^{p-1}, \quad x \geq \bar{R}_{4} .
\end{aligned}
$$

Let $R_{4}=\max \left\{2 R_{3}, \bar{R}_{4}\right\}$ and $\Omega_{4}=\left\{(u, v, w) \in Y:\|(u, v, w)\|_{Y}<R_{4}\right\}$. For any $(u, v, w) \in P \cap \partial \Omega_{4}$ and $t \in[1, e]$, we have

$$
\begin{array}{ll}
f(t, u(t), v(t), w(t)) \leq f^{\star}\left(t,\|(u, v, w)\|_{Y}\right), & t \in[1, e], \\
g(t, u(t), v(t), w(t)) \leq g^{\star}\left(t,\|(u, v, w)\|_{Y}\right), & t \in[1, e], \\
h(t, u(t), v(t), w(t)) \leq h^{\star}\left(t,\|(u, v, w)\|_{Y}\right), & t \in[1, e],
\end{array}
$$

then

$$
\begin{aligned}
Q_{1}(u, v)(t) & \leq \lambda \int_{1}^{e} G_{1}(e, s)\left(\int_{1}^{e} H_{1}(s, \tau) f^{\star}\left(\tau,\|(u, v, w)\|_{Y}\right) \frac{d \tau}{\tau}\right)^{\frac{1}{p-1}} \frac{d s}{s} \\
& \leq \lambda \int_{1}^{e} G_{1}(e, s)\left(\int_{1}^{e} H_{1}(\tau, \tau)\left(f_{\infty}^{s}+\epsilon\right)\left(\|(u, v, w)\|_{Y}\right)^{p-1} \frac{d \tau}{\tau}\right)^{\frac{1}{p-1}} \frac{d s}{s} \\
& =\lambda\left(f_{\infty}^{s}+\epsilon\right)^{\frac{1}{p-1}} \int_{1}^{e} G_{1}(e, s)\left(\int_{1}^{e} H_{1}(\tau, \tau) \frac{d \tau}{\tau}\right)^{\frac{1}{p-1}} \frac{d s}{s}\|(u, v, w)\|_{Y} \\
& \leq \rho_{1}\|(u, v, w)\|_{Y},
\end{aligned}
$$

so $\left\|Q_{1}(u, v, w)\right\| \leq \rho_{1}\|(u, v, w)\|_{Y},(u, v, w) \in P \cap \partial \Omega_{4}$. In a similar manner, we deduce $\left\|Q_{2}(u, v, w)\right\| \leq \rho_{2}\|(u, v, w)\|_{Y},\left\|Q_{3}(u, v, w)\right\| \leq \rho_{3}\|(u, v, w)\|_{Y}$. Therefore,

$$
\begin{aligned}
\|Q(u, v, w)\|_{Y} & =\left\|\left(Q_{1}(u, v, w), Q_{2}(u, v, w), Q_{3}(u, v, w)\right)\right\|_{Y} \\
& =\left\|Q_{1}(u, v, w)\right\|+\left\|Q_{2}(u, v, w)\right\|+\left\|Q_{3}(u, v, w)\right\| \\
& \leq \rho_{1}\|(u, v, w)\|_{Y}+\rho_{2}\|(u, v, w)\|_{Y}+\rho_{3}\|(u, v, w)\|_{Y} \\
& =\left(\rho_{1}+\rho_{2}+\rho_{3}\right)\|(u, v, w)\|_{Y} \\
& =\|(u, v, w)\|_{Y} .
\end{aligned}
$$

Hence, $\|Q(u, v, w)\|_{Y} \leq\|(u, v, w)\|_{Y}$. Define the set

$$
\Omega_{4}=\left\{(u, v, w) \in Y:\|(u, v, w)\|_{Y}<R_{4}\right\}
$$


then

$$
\|Q(u, v, w)\|_{Y} \leq\|(u, v, w)\|_{Y}, \quad \text { for }(u, v, w) \in P \cap \partial \Omega_{4} .
$$

Therefore, by (14), (15) and Theorem 3.5, we conclude that $Q$ has at least one fixed point $(u, v, w) \in P \cap\left(\bar{\Omega}_{4} \backslash \Omega_{3}\right)$ with $R_{3} \leq\|(u, v, w)\|_{Y} \leq R_{4}$. The proofs of the cases (b)-(d) are similar to that of (a) and we shall omit them.

\section{Example}

Let us consider an example to illustrate the above result.

Here $n=m=l=3, \alpha_{1}=\alpha_{2}=\alpha_{3}=\frac{5}{2}, \beta_{1}=\beta_{2}=\beta_{3}=\frac{3}{2}, p_{1}=q_{1}=r_{1}=\frac{5}{4}, p_{2}=q_{2}=r_{2}=\frac{1}{2}$, $\mu_{1}=\frac{1}{2}, \lambda_{1}=\frac{1}{3}, \xi=\frac{3}{2}$. Let $p=2$, we consider the Hadamard fractional differential equations

$$
\begin{cases}{ }^{H} D_{1^{+}}^{3 / 2}\left(\phi_{p}\left({ }^{H} D_{1^{+}}^{5 / 2} u(t)\right)\right)=\lambda^{p-1} f(t, u(t), v(t), w(t)), & t \in(1, e), \\ { }^{H} D_{1^{3}}^{3 / 2}\left(\phi_{p}\left({ }^{H} D_{1^{+}}^{5 / 2} v(t)\right)\right)=\mu^{p-1} g(t, u(t), v(t), w(t)), & t \in(1, e), \\ { }^{H} D_{1^{+}}^{3 / 2}\left(\phi_{p}\left({ }^{H} D_{1^{+}}^{5 / 2} w(t)\right)\right)=v^{p-1} h(t, u(t), v(t), w(t)), & t \in(1, e),\end{cases}
$$

with the three-point boundary conditions

$$
\left\{\begin{array}{l}
u(1)=u^{\prime}(1)=0, \quad(1 / 2) u^{5 / 4}(e)=(1 / 3) u^{5 / 4}(3 / 2), \\
\phi_{p}\left({ }^{H} D_{1^{+}}^{5 / 2} u(1)\right)=0=\left({ }^{H} D_{1^{+}}^{1 / 2}\left(\phi_{p}\left({ }^{H} D_{1^{+}}^{5 / 2} u(e)\right)\right)\right), \\
v(1)=v^{\prime}(1)=0, \quad(1 / 2) v^{5 / 4}(e)=(1 / 3) v^{5 / 4}(3 / 2), \\
\phi_{p}\left({ }^{H} D_{1^{+}}^{5 / 2} v(1)\right)=0=\left({ }^{H} D_{1^{+}}^{1 / 2}\left(\phi_{p}\left({ }^{H} D_{1^{+}}^{5 / 2} v(e)\right)\right)\right), \\
w(1)=w^{\prime}(1)=0, \quad(1 / 2) w^{5 / 4}(e)=(1 / 3) w^{5 / 4}(3 / 2), \\
\phi_{p}\left({ }^{H} D_{1^{+}}^{5 / 2} w(1)\right)=0=\left({ }^{H} D_{1^{+}}^{1 / 2}\left(\phi_{p}\left({ }^{H} D_{1^{+}}^{5 / 2} w(e)\right)\right)\right),
\end{array}\right.
$$

where

$$
\begin{aligned}
& f(t, u, v, w)=\frac{(\ln t+1)[800(u+v+w)+1](u+v+w)(4+\sin v)}{u+v+w+1}, \\
& g(t, u, v, w)=\frac{(\sqrt{\ln t+1})[400(u+v+w)+1](u+v+w)(3+\cos w)}{u+v+w+1}, \\
& h(t, u, v, w)=\frac{(\ln t+2)[200(u+v+w)+1](u+v+w)(2+\sin u)}{u+v+w+1} .
\end{aligned}
$$

After simple calculations, we get

$$
\begin{aligned}
& f_{0}^{s}=8, \quad f_{0}^{i}=5, \quad f_{\infty}^{s}=8000, \quad f_{\infty}^{i}=3000, \\
& g_{0}^{s}=5.6569, \quad g_{0}^{i}=4.4722, \quad g_{\infty}^{s}=37085, \quad g_{\infty}^{i}=894.43, \\
& h_{0}^{s}=6, \quad h_{0}^{i}=4.5, \quad h_{\infty}^{s}=1800, \quad h_{\infty}^{i}=450, \quad m=0.125, \\
& L_{1}=0.05903556919 \sigma_{1}, \quad L_{2}=0.1282828756 \sigma_{1}, \quad L_{3}=0.08446316582 \sigma_{2}, \\
& L_{4}=0.1195672885 \sigma_{2}, \quad L_{5}=0.094532722 \sigma_{3}, \quad L_{6}=0.1076892453 \sigma_{3},
\end{aligned}
$$

where $\sigma_{1}, \sigma_{2}, \sigma_{3}>0$ are three positive real numbers such that $\sigma_{1}+\sigma_{2}+\sigma_{3}=1$. 
Employing Theorem 4.2 of (a), for each $\lambda \in\left(L_{1}, L_{2}\right), \mu \in\left(L_{3}, L_{4}\right)$ and $v \in\left(L_{5}, L_{6}\right)$, there exists a positive solution $(u(t), v(t), w(t))$ of the Hadamard fractional differential equation (16)-(17).

\section{Acknowledgements}

The author wishes to thank the anonymous referees for their valuable suggestions.

\section{Funding}

Not applicable.

\section{Abbreviations}

Not applicable.

\section{Availability of data and materials}

Not applicable.

\section{Competing interests}

The author declares to have no competing interests.

\section{Authors' contributions}

The author was the only one to contribute in writing this article. The author read and approved the final manuscript.

\section{Publisher's Note}

Springer Nature remains neutral with regard to jurisdictional claims in published maps and institutional affiliations.

Received: 11 March 2019 Accepted: 20 May 2019 Published online: 29 May 2019

\section{References}

1. Kilbas, A.A., Srivastava, H.M., Trujillo, J.J.: Theory and Applications of Fractional Differential Equations. Elsevier, Amsterdam (2006)

2. Podlubny, I.: Fractional Differential Equations. Academic Press, New York (1999)

3. Miller, K.S., Ross, B.: An Introduction to the Fractional Calculus and Fractional Differential Equations. Wiley, New York (1993)

4. Sabatier, J., Agrawal, O.P., Machado, J.T.: Advances in Fractional Calculus, Theoretical Developments and Applications in Physics and Engineering. Springer, Dordrecht (2007)

5. Papageorglou, N.S., Radulescu, V.D., Repovs, D.D.: Nonlinear Analysis—Theory and Methods. Springer Monographs in Mathematics. Springer, Cham (2019)

6. Denton, Z., Ramirez, J.D.: Existence of minimal and maximal to RL fractional integro-differential initial value problems. Opusc. Math. 37(5), 705-724 (2017)

7. Dao, N.A., Diaz, J.I., Nguyen, Q.H.: Generalized Gagliardo-Nirenberg inequalities using Lorentz spaces, BMO, Hölder spaces and fractional Sobolev spaces. Nonlinear Anal. 173, 146-153 (2018)

8. Hao, X., Zhang, L.: Positive solutions of higher order fractional integral boundary value problem with a parameter. Nonlinear Anal., Model. Control 24(2), 210-223 (2019)

9. Hao, X., Zhang, L.: Positive solutions of a fractional thermostat model with a parameter. Symmetry. 11(1), 122 (2019). https://doi.org/10.3390/sym11010122

10. Hao, X., Sun, H., Liu, L.: Existence results for fractional integral boundary value problem involving fractional derivatives on an infinite interval. Math. Methods Appl. Sci. 41(16), 6984-6996 (2018)

11. Pu, R., Zhang, X., Cui, Y., Li, P., Wang, W.: Positive solutions for singular semipositone fractional differential equation subject to multipoint boundary conditions. J. Funct. Spaces 2017, Article ID 5892616 (2017)

12. Sun, Y., Zhang, X.: Existence and nonexistence of positive solutions for fractional-order two-point boundary value problems. Adv. Differ. Equ. 2014, 53 (2014)

13. Zhang, X., Zhong, Q.: Uniqueness of solution for higher-order fractional differential equations with conjugate type integral conditions. Fract. Calc. Appl. Anal. 20(6), 1471-1484 (2017)

14. Zhang, X., Zhong, Q:. Triple positive solutions for nonlocal fractional differential equations with singularities both on time and space variables. Appl. Math. Lett. 80, 12-19 (2018)

15. Lyons, J., Neugebauer, J.: Positive solutions of a singular fractional boundary value problem with a fractional boundary condition. Opusc. Math. 37(3), 421-434 (2017)

16. Hao, X., Wang, H.: Positive solutions of semipositone singular fractional differential systems with a parameter and integral boundary conditions. Open Math. 16, 581-596 (2018)

17. Rao, S.N.: Multiple positive solutions for a system of Riemann-Liouville fractional order two-point boundary value problems. Panam. Math. J. 25(1), 66-81 (2015)

18. Rao, S.N.: Solvability for a system of nonlinear fractional higher order three point boundary value problem. Fract. Differ. Calc. 7(2), 151-167 (2017)

19. Rao, S.N., Zico, M.: Positive solutions for a coupled system of nonlinear semipositone fractional boundary value problems. Int. J. Differ. Equ. 2019, Article ID 2893857 (2019)

20. Rao, S.N., Alesemi, M.: On a coupled system of fractional differential equations with nonlocal non-separated boundary conditions. Adv. Differ. Equ. 2019, 97 (2019)

21. Luca, R.: Positive solutions for a system of Riemann-Liouville fractional differential equations with multi-point fractional boundary conditions. Bound. Value Probl. 2017, 102 (2017) 
22. Henderson, J., Luca, R.: Positive solutions for a system of second-order multi-point boundary value problems. Appl. Math. Comput. 218(10), 6083-6094 (2012)

23. Henderson, J., Luca, R.: Boundary Value Problems for Systems of Differential, Difference and Fractional Equations: Positive Solutions. Elsevier, Amsterdam (2016)

24. Leibenson, L.S.: General problem of the movement of a compressible fluid in a porous medium. IzV. Akad. Nauk SSSR, Ser. Geogr. Geofiz. 9, 7-10 (1945)

25. Lu, H., Han, Z., Sun, S., Liu, J.: Existence on positive solution for boundary value problems of nonlinear fractional differential equations with p-Laplacian. Adv. Differ. Equ. 2013, 30 (2013)

26. Yan, F., Zuo, M., Hao, X.: Positive solution for a fractional singular boundary value problem with $p$-Laplacian operator. Bound. Value Probl. 2018, 51 (2018)

27. $\mathrm{Xu}$, J., Dong, W.: Existence and uniqueness of positive solutions for a fractional boundary value problem with p-Laplacian operator. Acta Math. Sinica (Chin. Ser.) 59, 385-396 (2016)

28. Zhang, X., Shao, Z., Zhong, Q., Zhao, Z.: Triple positive solutions for semipositone fractional differential equations $m$-point boundary value problems with singularities and p-q-order derivatives. Nonlinear Anal., Model. Control 23(6), 889-903 (2018)

29. Xu, J., O'Regan, D.: Positive solutions for a fractional $p$-Laplacian boundary value problem. Filomat 31(6), 1549-1558 (2017)

30. Rao, S.N.: Multiplicity of positive solutions for fractional differential equation with $p$-Laplacian boundary value problems. Int. J. Differ. Equ. 2016, Article ID 6906049 (2016)

31. Dong, X., Bai, Z., Zhang, S.: Positive solutions to boundary value problems of $p$-Laplacian with fractional derivative. Bound. Value Probl. 2017, 5 (2017)

32. Tian, Y., Wei, Y., Sun, S.: Multiplicity for fractional differential equations with $p$-Laplacian. Bound. Value Probl. 2018, 127 (2018)

33. He, J., Song, X.: The uniqueness of solution for a class of fractional order nonlinear systems with $p$-Laplacian operator Abstr. Appl. Anal. 2014, Article ID 921209 (2014)

34. Hao, X., Wang, H., Liu, L., Cui, Y.: Posive solutions for a system of nonlinear fractional nonlocal boundary value problems with parameters and p-Laplacian operator. Bound. Value Probl. 2017, 182 (2017)

35. Liu, Y., Xie, D., Bai, C., Yang, D.: Multiple positive solutions for a coupled system of fractional multi-point BVP with p-Laplacian operator. Adv. Differ. Equ. 2017, 168 (2017)

36. Rao, S.N.: Solvability of positive solutions for a systems of nonlinear fractional order BVPs with p-Laplacian. Adv. Pure Appl. Math. 10(2), 141-153 (2019)

37. Rao, S.N.: Multiple positive solutions for coupled system of $p$-Laplacian fractional order three-point boundary value problems. Rocky Mt. J. Math. (2019, in press). https://projecteuclid.org/euclid.rmjm/1540281667

38. Rao, S.N.: Multiplicity of positive solutions for coupled system of fractional differential equation with $p$-Laplacian two-point BVPs. J. Appl. Math. Comput. 55, 41-58 (2017)

39. Rao, S.N., Alesemi, A.: Existence of positive solutions for a systems of nonlinear fractional differential equation with p-Laplacian. Asian-Eur. J. Math. (2019, in press). https://doi.org/10.1142/S1793557120500898

40. Hadamard, J.: Essai sur letude des fonctions donnees par leur developpement de Taylor. J. Math. Pures Appl. 8, $101-186$ (1892)

41. Wang, J., Zhang, Y.: On the concept and existence of solutions for fractional impulsive systems with Hadamard derivatives. Appl. Math. Lett. 39, 85-90 (2015)

42. Huang, H., Liu, W.: Positive solutions for a class of nonlinear Hadamard fractional differential equations with a paprameter. Adv. Differ. Equ. 2018, 96 (2018)

43. Wang, G.T., Pei, K., Agarwal, R.P., Zhang, L.H., Ahmad, B.: Nonlocal Hadamard fractional boundary value problem with Hadamard integral and discrete boundary conditions on a half-line. J. Comput. Appl. Math. 343, 230-239 (2018)

44. Zhai, C., Wang, W., Li, H.: A uniqueness method to a new Hadamard fractional differential system with four-point boundary conditions. J. Inequal. Appl. 2018, 207 (2018)

45. Yang, W.: Positive solutions for singular coupled integral boundary value problems of nonlinear Hadamard fractional differential equations. J. Nonlinear Sci. Appl. 8, 110-129 (2015)

46. Li, Y.L., Lin, S.Y.: Positive solution for the nonlnear Hadamard type fractional differential equation with $p$-Laplacian J. Funct. Spaces Appl. 2013, Article ID 951643 (2013)

47. Wang, G., Wang, T.: On a nonlinear Hadamard type fractional differential equation with $p$-Laplacian operator and strip condition. J. Nonlinear Sci. Appl. 9, 5073-5081 (2016)

48. Zhang, K., Wang, J., Ma, W.: Solutions for integral boundary-value problems of nonlinear Hadamard fractional differential equations. J. Funct. Spaces 2018, Article ID 2193234 (2018)

49. Guo, D.J., Lakshmikantham, V.: Nonlinear Problems in Abstract Cones. Academic Press, Boston (1988)

50. Krasnosel'skii, M.A.: Positive Solutions of Operator Equations. Noordhoff, Groningen (1964) 